\title{
Nontargeted Metabolomic Analysis of Four Different Parts of Platycodon grandiflorum Grown in Northeast China
}

\author{
Cuizhu Wang ${ }^{1,2}$, Nanqi Zhang ${ }^{1,2}$, Zhenzhou Wang ${ }^{1,2}$, Zeng Qi ${ }^{1,2}$, Hailin Zhu ${ }^{1,2}$, \\ Bingzhen Zheng ${ }^{1,2}$, Pingya $\mathrm{Li}^{1,2, *}$ and Jinping Liu ${ }^{1,2, *}$ ib \\ 1 School of Pharmaceutical Sciences, Jilin University, Fujin Road 1266, Changchun 130021, China; \\ wangcz15@mails.jlu.edu.cn (C.W.); zhangnq15@mails.jlu.edu.cn (N.Z.);wzz16@mails.jlu.edu.cn (Z.W.); \\ qizeng15@mails.jlu.edu.cn (Z.Q.); zhuhl14@mails.jlu.edu.cn (H.Z.); zhengbz15@mails.jlu.edu.cn (B.Z.) \\ 2 National and Local Joint Engineering Research Center for Ginseng Innovative Drugs Development, \\ Western Chaoyang Road 45, Changchun 130021, China \\ * $\quad$ Correspondence: lipy@jlu.edu.cn (P.L.); liujp@jlu.edu.cn (J.L.); Tel.: +86-431-8561-9803 (P.L. \& J.L.)
}

Received: 5 July 2017; Accepted: 29 July 2017; Published: 3 August 2017

\begin{abstract}
Platycodonis radix is extensively used for treating cough, excessive phlegm, sore throat, bronchitis and asthma in the clinic. Meanwhile, the stems, leaves and seeds of Platycodon grandiflorum (PG) have some pharmaceutical activities such as anti-inflammation and anti-oxidation effects, etc. These effects must be caused by the different metabolites in various parts of herb. In order to profile the different parts of $\mathrm{PG}$, the ultra-high performance liquid chromatography combined with quadrupole time-of- flight mass spectrometry (UPLC-QTOF-MS ${ }^{\mathrm{E}}$ ) coupled with UNIFI platform and multivariate statistical analyses was used in this study. Consequently, for the constituent screening, $73,42,35,44$ compounds were characterized from the root, stem, leaf and seed, respectively. The stem, leaf and seed contain more flavonoids but few saponins that can be easily discriminated in the root. For the metabolomic analysis, 15, 5, 7, 11 robust biomarkers enabling the differentiation among root, stem, leaf and seed, were discovered. These biomarkers can be used for rapid identification of four different parts of PG grown in northeast China.
\end{abstract}

Keywords: Platycodon grandiflorum; nontargeted metabolomic analysis; different part; UPLC-QTOF-MS ${ }^{\mathrm{E}}$

\section{Introduction}

It is well-known that there are both chemical and pharmacological differences in different parts of herbs. Taking Aristolochia mollissima Hance as an example, the fruits are used to treat cough and asthma, the roots have obvious antihypertensive effects, while the stems and leaves are rheumatoid medicines. This phenomenon also exists in other herbs, such as Lycium barbarum, Polygonum Multiflorum Thunb., Trichosanthes kirilowii Maxim, Ephedra sinice Stapf, etc. [1].

As both food and medicine, Platycodon grandiflorum (Jacq.) A. DC. (PG) is known as "Jiegeng" in China, "Huridunzhaga" in Mongolia, "Kikyo" in Japan and "Doraji" in North Korea [2]. In clinical, the root of PG which has various biological activities, such as apophlegmatic and antitussive [3], anti-inflammation [4], immunoregulation [5], anti-oxidant [6], etc., has been widely used for the treatment of cough, excessive phlegm, and sore throat. In addition, the stem and leaf of PG also have anti-inflammatory [7] and anti-oxidant [8,9] activities, while research on the pharmacological effects of PG seed is currently non-existent.

PG is a rich source of different natural products with various structural patterns. Around 100 compounds have been isolated from the roots of PG, including steroidal saponins, flavonoids, phenolic acids, polyacetylenes, sterols, etc. [2]. Triterpenoid saponins, mainly of the oleanane family 
pentacyclic type, are the active components of the root of PG [10]. Several flavonoids and phenolic acids were isolated from the aerial parts of PG [11]. Two glycosides and four flavonoids were isolated from the seeds of PG [12]. Recently, instead of traditional separation and identification method, a combination of ultra-high performance liquid chromatography (UHPLC) separation, quadrupole time-of-flight tandem mass spectrometry (QTOF-MS/MS) detection and automated data processing software UNIFI with scientific library was innovatively used for screening and identifying chemical components in herbal medicines [13,14] and traditional Chinese medicine formulas [15]. In 2015, Lee et al. reported the global profiling of various metabolites in PG by UPLC-QTOF/MS [16]. In that paper, a total of 20 metabolites were characterized from the roots, and 56 compounds from stems and leaves of PG grown in Korea. Herbs collected from different regions will show certain differences both in chemical constituents and in pharmacological activities [17]. For example, saponins in the root of PG from different sites in Gyeongnam Province, Korea showed different contents [18]. The ${ }^{1} \mathrm{H}$-NMR-based metabolomics with OPLS-DA statistical models was used to cluster the ginseng samples from Korea and China, and the result suggested that the chemical profiles from two countries are quite different due to their different geographical origins [19]. Hence, in order to illustrate different chemical constituents from the different regions and from the different parts of the plants, and to better clarify the pharmacological fundamental substances of PG, the root, stem, leaf and seed of PG produced in Jilin Province, China were taken as samples in this paper.

Metabolomics, including targeted and untargeted complementary approaches, is primarily concerned with identification and quantitation of small-molecule metabolites $(<1500 \mathrm{Da})$ [20]. Recently, because of its ability to profile diverse classes of metabolites, untargeted metabolomics has been widely used to compare the overall metabolic composition of different samples [21]. An untargeted analysis approach is mainly applied in metabolite identification through mass-based search followed by manual verification [20] Being a sensitive, efficient, reliable, accurate and nondestructive method, UPLC-QTOF-MS has been widely used recently in this kind of analysis, such as exploring the early detection of mycotoxins in wheat [22], estimating compliance to a dietary pattern [23], exploring the bioavailability of the secoiridoids from a seed/fruit extract in human healthy volunteers [24], evaluating the enantioselective metabolic perturbations in MCF-7 cells after treatment with $R$-metalaxyl and S-metalaxyl [25].

In this study we focus on both the quickly chemical components' screening and the non-targeted metabolomic analysis of the root, stem, leaf and seed of PG. UPLC-QTOF-MS ${ }^{\mathrm{E}}$, UNIFI platform and multivariate statistical analyses, such as principal component analysis (PCA) and orthogonal partial least squares discriminant analysis (OPLS-DA) were used to profile the four different plant parts and to find the biomarkers among these four parts of PG grown in northeast China.

\section{Results}

\subsection{Identification of Components from Different Parts of PG}

As a result, a total of 159 compounds were identified or tentatively characterized in both positive and negative mode from the four parts of PG, the base peak intensity (BPI) chromatograms are shown in Figure 1, and their chemical structures are shown in Figure 2. More specifically, 73, 42, 35, 44 compounds were characterized from the root, stem, leaf and seed respectively (Table 1), including triterpenoid saponins, organic acids, steroids, phenols, flavonoids, alcohols, amino acids, coumarins, terpenoids, alkaloids and amides and so on.

For the compounds which have isomers, they may be distinguished by their characteristic MS fragmentation patterns reported in literature, or may be compared with the retention times of reference standards. Taking compounds 98 and 106 as example, both have the same protonated ion $[\mathrm{M}+\mathrm{H}]^{+}$at $m / z 1413.6530$ and 1413.6530 . In the results, they matched $3 "-O-$-acetylpolygalacin D2 and 2"-O-acetylpolygalacin D2, respectively. 


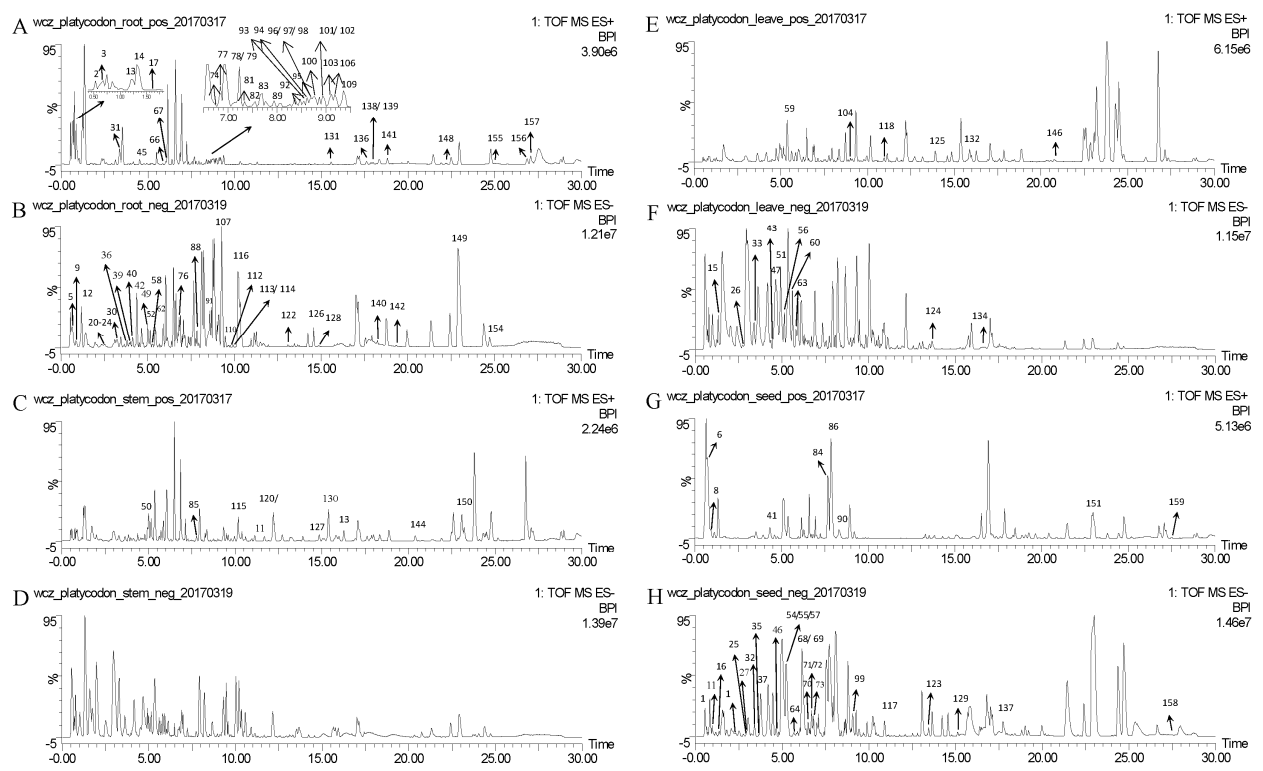

Figure 1. The representative base peak intensity (BPI) chromatograms of root in positive (A) and negative (B) modes; of stem in positive (C) and negative (D) modes; of leaf in positive (E) and negative (F) modes; of seed in positive $(\mathbf{G})$ and negative $(\mathbf{H})$ modes.

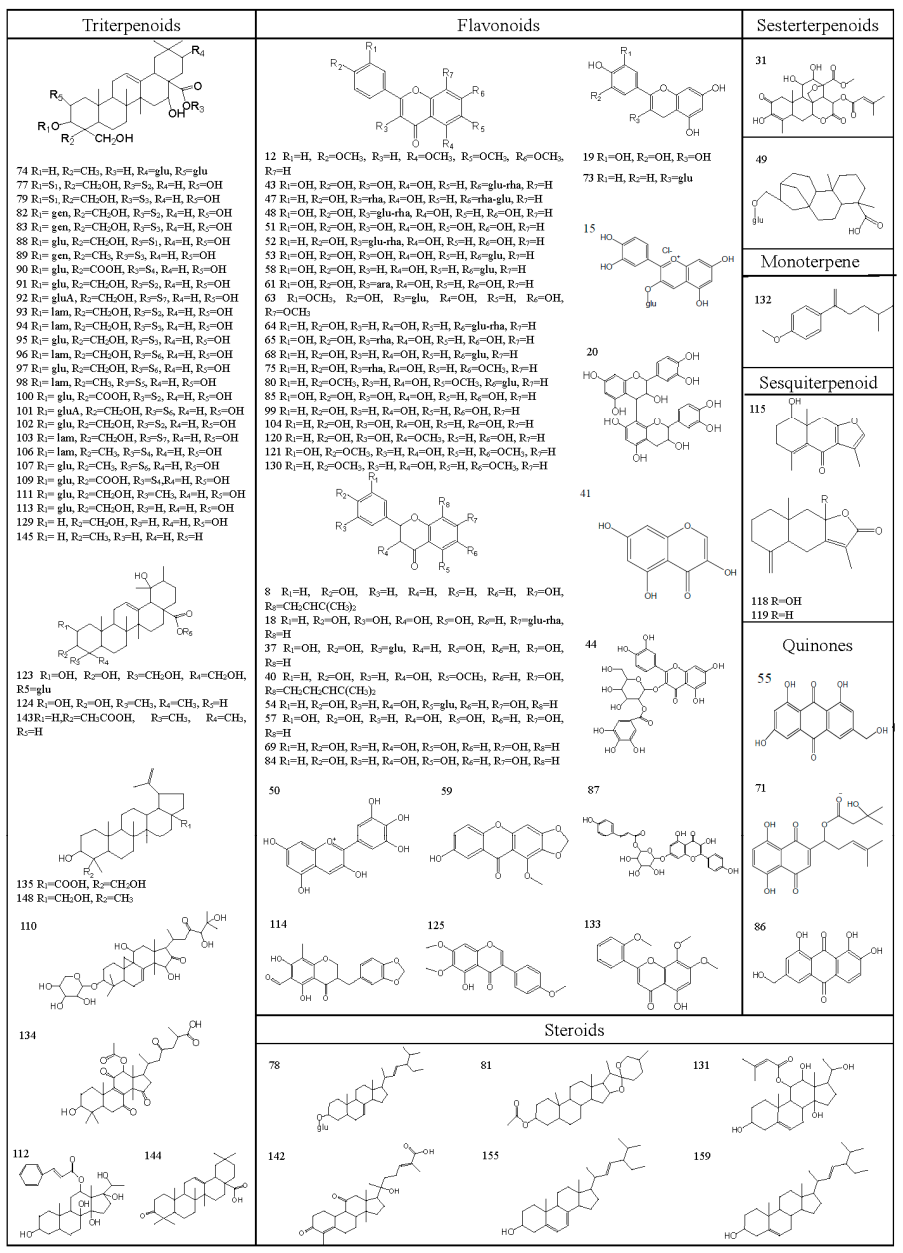

Figure 2. Cont. 


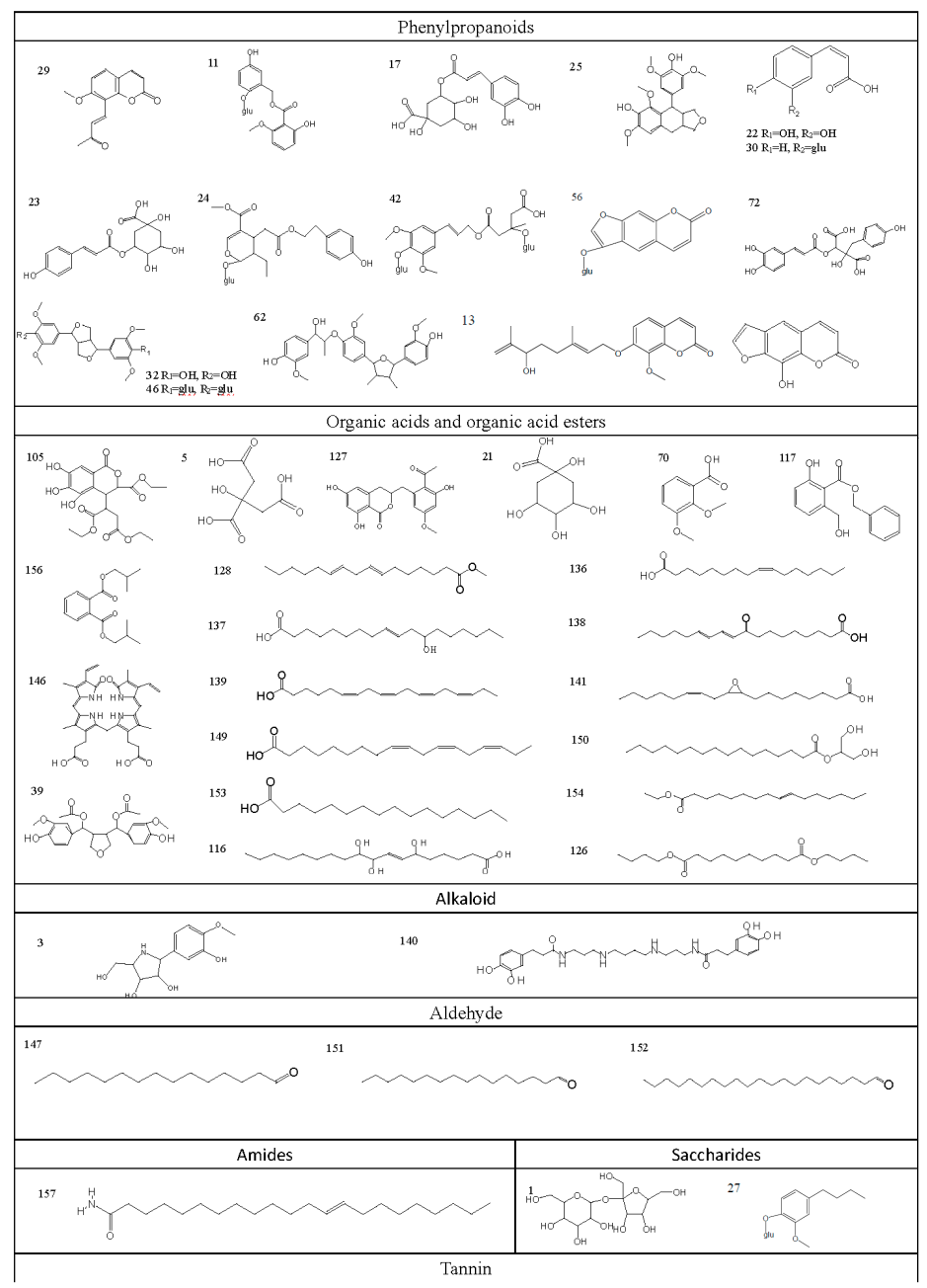

$38 \mathrm{R}=\mathrm{A}$

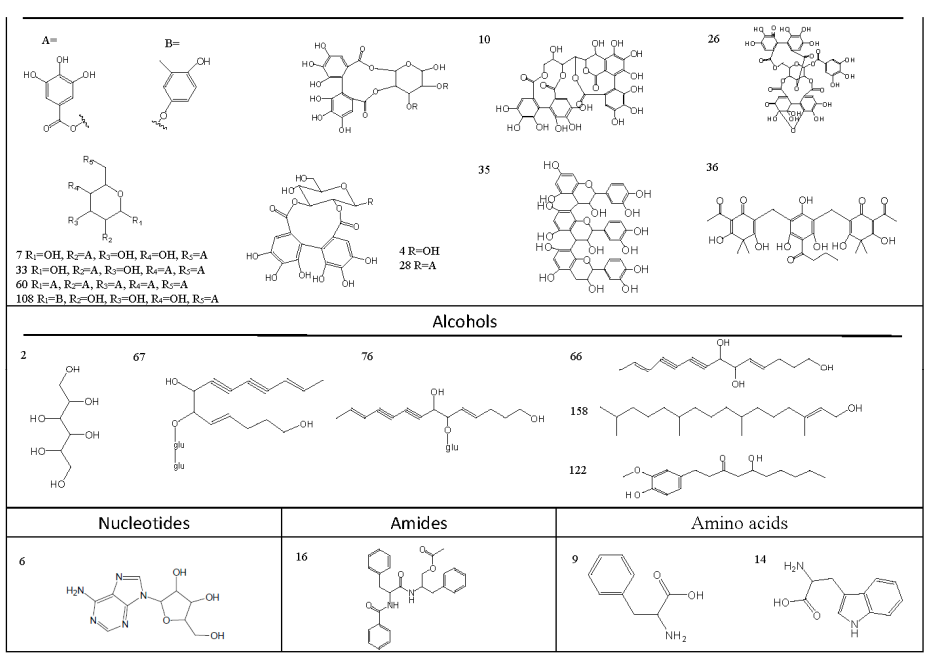

Figure 2. Chemical structures of compounds identified in PG. 
Table 1. Compounds identified from different parts of PG by UPLC-QTOF-MS ${ }^{\mathrm{E}}$.

\begin{tabular}{|c|c|c|c|c|c|c|c|c|c|}
\hline No. & $\begin{array}{c}t_{\mathrm{R}} \\
(\mathrm{min})\end{array}$ & Formula & $\begin{array}{c}\text { Experimental } \\
\text { (Da) }\end{array}$ & $\begin{array}{l}\text { Theoretical } \\
\text { (Da) }\end{array}$ & $\begin{array}{c}\text { Mass Error } \\
(\mathrm{ppm})\end{array}$ & Adducts & $\mathrm{MS}^{\mathrm{E}}$ Fragmentation & Component Name & Source \\
\hline $1^{*}$ & 0.59 & $\mathrm{C}_{12} \mathrm{H}_{22} \mathrm{O}_{11}$ & 342.1169 & 342.1162 & 2.04 & $-\mathrm{H}$ & $323.0984,195.0510, \mathbf{1 6 1 . 0 4 6 5}$ & Sucrose & $\mathrm{D}$ \\
\hline $2 *$ & 0.60 & $\mathrm{C}_{6} \mathrm{H}_{14} \mathrm{O}_{6}$ & 182.0797 & 182.0790 & 3.04 & $+\mathrm{Na}$ & $205.0689, \mathbf{1 5 2 . 0 7 1 3}$ & Mannitol & $\mathrm{R}$ \\
\hline 3 & 0.67 & $\mathrm{C}_{12} \mathrm{H}_{17} \mathrm{NO}_{5}$ & 255.1114 & 255.1107 & 2.91 & $+\mathrm{H}$ & $256.1114, \mathbf{2 2 6 . 1 0 7 4 , 1 2 2 . 0 3 7 5}$ & Radicamine A & $\mathrm{R}$ \\
\hline 4 & 0.68 & $\mathrm{C}_{20} \mathrm{H}_{18} \mathrm{O}_{14}$ & 482.0682 & 482.0697 & -2.95 & $-\mathrm{H}$ & $343.0676,301.0007,274.0119,191.0554,152.0124$ & 2,3-(S)-Hexahydroxydiphenoyl-D-glucose a & $\mathrm{S}, \mathrm{L}$ \\
\hline $5^{*}$ & 0.71 & $\mathrm{C}_{6} \mathrm{H}_{8} \mathrm{O}_{7}$ & 192.0278 & 192.0270 & 3.93 & $-\mathrm{H}$ & $191.0205, \mathbf{1 7 3 . 0 0 7 7}, 111.0089$ & Citric acid & $\mathrm{R}$ \\
\hline 6* & 0.75 & $\mathrm{C}_{10} \mathrm{H}_{13} \mathrm{~N}_{5} \mathrm{O}_{4}$ & 267.0974 & 267.0968 & 2.23 & $+\mathrm{H}$ & 218.1020, 136.0634 & Adenosine & $\mathrm{D}$ \\
\hline 7 & 0.82 & $\mathrm{C}_{20} \mathrm{H}_{20} \mathrm{O}_{14}$ & 484.0857 & 484.0853 & 0.78 & $-\mathrm{H}$ & $313.0568,183.0308, \mathbf{1 6 9 . 0 1 5 6 , 1 5 2 . 0 1 2 3}$ & 2,6 -Di-O-Galloyl- $\beta$-D-glucose ${ }^{\text {a }}$ & $\mathrm{S}, \mathrm{L}$ \\
\hline 8 & 0.85 & $\mathrm{C}_{20} \mathrm{H}_{20} \mathrm{O}_{4}$ & 324.1347 & 324.1362 & -4.38 & $+\mathrm{H}$ & 203.0708, 175.0758, 164.0463, 149.0602, 103.0556 & Isobavachin a & $\mathrm{D}$ \\
\hline $9 *$ & 0.86 & $\mathrm{C}_{9} \mathrm{H}_{11} \mathrm{NO}_{2}$ & 165.0796 & 165.0790 & 3.98 & $-\mathrm{H}$ & $164.0724,147.0456,103.0549$ & Phenylalanine & $\mathrm{R}$ \\
\hline 10 & 0.95 & $\mathrm{C}_{34} \mathrm{H}_{24} \mathrm{O}_{22}$ & 784.0751 & 784.0759 & -1.05 & $-\mathrm{H}$ & $421.0417,337.0214,249.0416,182.0223,168.0074,149.9967$ & Casuariin $^{\mathrm{a}}$ & $\mathrm{s}$ \\
\hline 11 & 0.97 & $\mathrm{C}_{21} \mathrm{H}_{24} \mathrm{O}_{11}$ & 452.1341 & 452.1319 & 4.86 & $-\mathrm{H}$ & $299.0771,289.0737,271.0611,165.0206,137.0257$ & Curculigoside $\mathrm{B}^{\mathrm{a}}$ & $\mathrm{D}$ \\
\hline 12 & 1.02 & $\mathrm{C}_{19} \mathrm{H}_{18} \mathrm{O}_{6}$ & 342.1089 & 342.1103 & -4.14 & $-\mathrm{H}$ & $211.0628,181.0506,179.0349,161.0240,151.0404$ & $5,6,7,4^{\prime}$-Tetramethoxyflavone a & $\mathrm{R}$ \\
\hline 13 & 1.24 & $\mathrm{C}_{20} \mathrm{H}_{24} \mathrm{O}_{5}$ & 344.1609 & 344.1624 & -3.98 & $+\mathrm{Na}$ & $222.0916,194.0973,182.0611,127.0394$ & Schininallylol a & $\mathrm{R}$ \\
\hline $14^{*}$ & 1.35 & $\mathrm{C}_{11} \mathrm{H}_{12} \mathrm{~N}_{2} \mathrm{O}_{2}$ & 204.0903 & 204.0899 & 2.29 & $+\mathrm{H}$ & 188.0706, $144.0808,132.0813,118.0661$ & Tryptophan & $\mathrm{R}$ \\
\hline 15 & 1.36 & $\mathrm{C}_{21} \mathrm{H}_{21} \mathrm{ClO}_{11}$ & 484.0775 & 484.0772 & 0.45 & $-\mathrm{H}$ & $309.0630,287.0594,124.0163, \mathbf{1 0 9 . 0 2 9 1}$ & Cyanidin 3-glucoside a & $\mathrm{L}$ \\
\hline 16 & 1.37 & $\mathrm{C}_{27} \mathrm{H}_{28} \mathrm{~N}_{2} \mathrm{O}_{4}$ & 444.2034 & 444.2049 & -3.41 & $-\mathrm{H}$ & $235.1215, \mathbf{1 7 5 . 0 6 2 6}, 173.0464,131.0364,105.0356$ & Aurantiamide acetate ${ }^{a}$ & $\mathrm{D}$ \\
\hline $17 *$ & 1.73 & $\mathrm{C}_{16} \mathrm{H}_{18} \mathrm{O}_{9}$ & 354.0950 & 354.0951 & -0.22 & $+\mathrm{H}$ & $192.0663, \mathbf{1 6 3 . 0 3 9 6}, 145.0294,135.0452$ & Chlorogenic acid & $\mathrm{R}$ \\
\hline 18 & 2.15 & $\mathrm{C}_{27} \mathrm{H}_{32} \mathrm{O}_{16}$ & 612.1712 & 612.1690 & 3.51 & $-\mathrm{H}$ & $593.1511,461.1313,303.0532,285.0428,177.0209,151.0052$ & $\begin{array}{l}(2 R, 3 R) \text {-Taxifolin7-O- } \alpha \text {-L-rhamnopyranosyl- }(1 \rightarrow 6) \text { - } \\
\beta \text {-D-glucopyranoside }\end{array}$ & $\mathrm{D}$ \\
\hline 19 & 2.30 & $\mathrm{C}_{30} \mathrm{H}_{26} \mathrm{O}_{12}$ & 578.1430 & 578.1424 & 0.98 & $-\mathrm{H}$ & $449.0876,425.0875,407.0777, \mathbf{2 8 9 . 0 7 1 8}, 125.0257$ & $\begin{array}{l}\text { P-Drocyanidin } B_{1} \text { a } \\
\text { Pro }\end{array}$ & $\mathrm{D}$ \\
\hline 20 * & 2.31 & $\mathrm{C}_{15} \mathrm{H}_{14} \mathrm{O}_{7}$ & 306.0738 & 306.0740 & -0.53 & $+\mathrm{HCOO}$ & $\mathbf{1 7 9 . 0 3 4 9 ,}, 167.0343,163.0406,161.0241,109.0315$ & Gallocatechin & $\mathrm{R}$ \\
\hline 21 * & 2.34 & $\mathrm{C}_{7} \mathrm{H}_{12} \mathrm{O}_{6}$ & 192.0637 & 192.0634 & 1.53 & $-\mathrm{H}$ & $173.0480, \mathbf{1 2 7 . 0 4 0 6 , 1 1 6 . 0 5 1 4 , 1 1 1 . 0 4 5 6}$ & Quinine acid & $\mathrm{R}$ \\
\hline 22 * & 2.35 & $\mathrm{C}_{9} \mathrm{H}_{8} \mathrm{O}_{4}$ & 180.0425 & 180.0423 & 1.60 & $-\mathrm{H}$ & $161.0241,135.0451, \mathbf{1 3 3 . 0 2 9 7}, 109.0315,108.0224$ & Caffeic acid & $\mathrm{R}$ \\
\hline 23 & 2.36 & $\mathrm{C}_{16} \mathrm{H}_{18} \mathrm{O}_{8}$ & 338.0993 & 338.1002 & -2.62 & $-\mathrm{H}$ & 191.0567, $177.0195,161.0243,119.0505,105.0351$ & 3-O-trans-Coumaroylquinic acid & $\mathrm{R}$ \\
\hline 24 & 2.70 & $\mathrm{C}_{25} \mathrm{H}_{34} \mathrm{O}_{12}$ & 526.2045 & 526.2050 & -1.04 & $-\mathrm{H}$ & 363.1452, 315.1244, $179.0713,167.0711,149.0612$ & LucidumosideA ${ }^{\text {a }}$ & $\mathrm{R}$ \\
\hline 25 & 2.78 & $\mathrm{C}_{22} \mathrm{H}_{26} \mathrm{O}_{7}$ & 402.1670 & 402.1679 & -1.88 & $+\mathrm{HCOO}$ & $327.0884, \mathbf{3 0 3 . 0 8 8 5}, 297.0421,209.0844,137.0256$ & Neociwujiaphenol a $^{{ }^{2}}$ & $\mathrm{D}$ \\
\hline 26 & 2.81 & $\mathrm{C}_{41} \mathrm{H}_{28} \mathrm{O}_{27}$ & 952.0809 & 952.0818 & -0.97 & $-\mathrm{H}$ & $605.0777,479.0469,481.0642,453.0677,246.0169$ & Geraniin $^{\text {a }}$ & $\mathrm{L}$ \\
\hline 27 & 2.98 & $\mathrm{C}_{17} \mathrm{H}_{26} \mathrm{O}_{7}$ & 342.1678 & 342.1679 & -0.01 & $+\mathrm{HCOO}$ & $\begin{array}{l}281.0651, \mathbf{1 6 3 . 1 1 3 0}, 121.0300 \\
\end{array}$ & Citrusin C & $\mathrm{D}$ \\
\hline 28 & 2.99 & $\mathrm{C}_{27} \mathrm{H}_{22} \mathrm{O}_{18}$ & 634.0813 & 634.0806 & 1.16 & $-\mathrm{H}$ & 601.0460, 463.0518, 419.0617, 301.0007, 291.0156, 275.0208 & Sanguiin $\mathrm{H}-4^{\text {a }}$ & $\mathrm{s}$ \\
\hline 29 & 3.05 & $\mathrm{C}_{14} \mathrm{H}_{12} \mathrm{O}_{4}$ & 244.0745 & 244.0736 & 3.14 & $+\mathrm{HCOO}$ & $203.0721, \mathbf{1 8 7 . 0 4 0 2}, 161.0250,123.0457,109.0303$ & cis-Osthenone & $\mathrm{D}$ \\
\hline 30 & 3.24 & $\mathrm{C}_{15} \mathrm{H}_{18} \mathrm{O}_{8}$ & 326.1003 & 326.1002 & 0.33 & $+\mathrm{HCOO}$ & $\mathbf{1 6 2 . 0 5 5 2 , 1 2 9 . 0 1 9 9 , 1 2 1 . 0 3 0 4}$ & 4-O- $\beta$-D-glucopyranosyl-trans-cinnamic acid a & $\mathrm{R}, \mathrm{D}$ \\
\hline 31 * & 3.28 & $\mathrm{C}_{26} \mathrm{H}_{32} \mathrm{O}_{11}$ & 520.1968 & 520.1945 & 4.46 & $+\mathrm{H}$ & $443.0984,341.1392,163.075$ & Brusatol & $\mathrm{R}$ \\
\hline 32 & 3.42 & $\mathrm{C}_{22} \mathrm{H}_{26} \mathrm{O}_{8}$ & 418.1631 & 418.1628 & 0.72 & $-\mathrm{H}$ & $359.1465,179.0726,164.0477,149.0251, \mathbf{1 2 5 . 0 2 5 4}$ & (+)-Syringaresinol & $\mathrm{D}$ \\
\hline 33 & 3.55 & $\mathrm{C}_{27} \mathrm{H}_{24} \mathrm{O}_{18}$ & 636.0969 & 636.0963 & 0.99 & $-\mathrm{H}$ & $483.0791,465.0679,331.0667,313.0578,169.0163$ & $2,4,6$-Tri- $O$-galloyl- $\beta$-D-glucose a & $\mathrm{S}, \mathrm{L}$ \\
\hline $34^{*}$ & 3.65 & $\mathrm{C}_{11} \mathrm{H}_{6} \mathrm{O}_{4}$ & 202.0260 & 202.0266 & -2.32 & $+\mathrm{HCOO}$ & 163.0419, 149.0244, 134.0373, 133.0304 & Xanthotoxol & $\mathrm{S}, \mathrm{L}$ \\
\hline 35 & 3.67 & $\mathrm{C}_{45} \mathrm{H}_{38} \mathrm{O}_{18}$ & 866.2079 & 866.2058 & 2.37 & $-\mathrm{H}$ & $575.1207,407.0781,289.0730,179.0356$ & Arecatannin $\mathrm{A}_{1}$ a & $\mathrm{D}$ \\
\hline 36 & 3.76 & $\mathrm{C}_{32} \mathrm{H}_{36} \mathrm{O}_{12}$ & 612.2223 & 612.2207 & 2.59 & $-\mathrm{H}$ & $562.1866,518.1583,210.0880,135.0462$ & Filixic acid ABA a & $\mathrm{R}$ \\
\hline 37 & 3.78 & $\mathrm{C}_{21} \mathrm{H}_{22} \mathrm{O}_{12}$ & 466.1128 & 466.1111 & 3.59 & $-\mathrm{H}$ & $285.0428,177.0208,165.0568,151.0053,137.0257,124.0178$ & Taxifolin-3-O-glucoside a & $\mathrm{D}$ \\
\hline 38 & 3.80 & $\mathrm{C}_{34} \mathrm{H}_{26} \mathrm{O}_{22}$ & 786.0915 & 786.0916 & -0.08 & $-\mathrm{H}$ & $615.0646,597.0511,445.0416,301.0021,125.0258$ & Collinin ${ }^{a}$ & $\mathrm{~s}$ \\
\hline 39 & 3.82 & $\mathrm{C}_{24} \mathrm{H}_{28} \mathrm{O}_{9}$ & 460.1739 & 460.1733 & 1.24 & $-\mathrm{H}$ & $414.1699,389.1244,193.0528, \mathbf{1 3 7 . 0 2 6 1}, 125.0258$ & Sanjidin $\mathrm{A}^{\mathrm{a}}$ & $\mathrm{R}$ \\
\hline 40 & 4.30 & $\mathrm{C}_{22} \mathrm{H}_{24} \mathrm{O}_{6}$ & 384.1560 & 384.1573 & -2.96 & $+\mathrm{HCOO}$ & 325.1065, 313.1078, 310.0838, 150.0322 & Sophoflavescenol a & $\mathrm{R}$ \\
\hline 41 & 4.33 & $\mathrm{C}_{9} \mathrm{H}_{6} \mathrm{O}_{5}$ & 194.0211 & 194.0215 & -2.35 & $+\mathrm{H}$ & $177.0183, \mathbf{1 5 3 . 0 1 7 8 ,} 138.0309,127.0398$ & 3,5,7-Trihydroxychromone & $\mathrm{D}$ \\
\hline 42 & 4.38 & $\mathrm{C}_{29} \mathrm{H}_{42} \mathrm{O}_{18}$ & 678.2395 & 678.2371 & 3.54 & $-\mathrm{H}$ & 497.1692, 453.1789, 323.0997, 291.1258, 161.0471 & TangshenosideI & $\mathrm{R}$ \\
\hline 43 & 4.48 & $\mathrm{C}_{27} \mathrm{H}_{30} \mathrm{O}_{16}$ & 610.1554 & 610.1534 & 3.24 & $-\mathrm{H}$ & 463.0844, 313.0580, 265.0370, $190.9983,151.0043$ & Quercetin-7-O-rutinoside & $\mathrm{L}$ \\
\hline 44 & 4.50 & $\mathrm{C}_{28} \mathrm{H}_{24} \mathrm{O}_{16}$ & 616.1084 & 616.1064 & 3.27 & $-\mathrm{H}$ & $313.0580,190.9983,177.0206, \mathbf{1 6 9 . 0 1 5 8 ,} 151.0043$ & $2^{\prime \prime}-\mathrm{O}$-Galloylhyperoside a & $\mathrm{S}, \mathrm{L}$ \\
\hline 45 & 4.53 & $\mathrm{C}_{11} \mathrm{H}_{12} \mathrm{O}_{3}$ & 192.0791 & 192.0786 & 2.21 & $+\mathrm{H}$ & $\begin{array}{l}193.0863,167.0703, \mathbf{1 6 1 . 0 6 0 3} \\
\end{array}$ & Myristicin & $\mathrm{R}$ \\
\hline
\end{tabular}


Table 1. Cont.

\begin{tabular}{|c|c|c|c|c|c|c|c|c|c|}
\hline No. & $\begin{array}{c}t_{\mathrm{R}} \\
(\mathrm{min})\end{array}$ & Formula & $\begin{array}{l}\text { Experimental } \\
\text { (Da) }\end{array}$ & $\begin{array}{l}\text { Theoretical } \\
\text { (Da) }\end{array}$ & $\begin{array}{c}\text { Mass Error } \\
(\mathrm{ppm})\end{array}$ & Adducts & $\mathrm{MS}^{\mathrm{E}}$ Fragmentation & Component Name & Source \\
\hline 46 & 4.66 & $\mathrm{C}_{34} \mathrm{H}_{46} \mathrm{O}_{18}$ & 742.2707 & 742.2684 & 2.90 & $+\mathrm{HCOO}$ & $579.2040, \mathbf{4 1 7 . 1 5 6 4 , 1 8 1 . 0 5 2 0 , 1 4 9 . 0 2 4 8}$ & Syringaresinol-di-O- $\beta$-D-glucoside $^{a}$ & $\mathrm{D}$ \\
\hline 47 & 4.72 & $\mathrm{C}_{33} \mathrm{H}_{40} \mathrm{O}_{19}$ & 740.2178 & 740.2164 & 1.92 & $-\mathrm{H}$ & $593.1506,575.1401,429.0824,335.0414, \mathbf{2 8 4 . 0 3 3 6}$ & Grosvenorine ${ }^{a}$ & $\mathrm{~S}, \mathrm{~L}$ \\
\hline $48^{*}$ & 4.93 & $\mathrm{C}_{27} \mathrm{H}_{30} \mathrm{O}_{16}$ & 610.1550 & 610.1534 & 2.59 & $-\mathrm{H}$ & 401.0912, 301.0365, 299.0205, 247.0609 & Rutin & S, L, D \\
\hline 49 & 4.94 & $\mathrm{C}_{26} \mathrm{H}_{42} \mathrm{O}_{8}$ & 482.2874 & 482.2880 & -1.13 & $+\mathrm{HCOO}$ & 261.1352,179.1074,149.0608, 125.0589 & 17-O- $\beta$-D-Glucopyra-nosyl-16 $\beta$-H-ent-kauran-19-oicacid a & $\mathrm{R}$ \\
\hline 50 * & 4.96 & $\mathrm{C}_{15} \mathrm{H}_{10} \mathrm{O}_{7}$ & 302.0427 & 302.0427 & 0.15 & $+\mathrm{H}$ & $161.0264, \mathbf{1 2 3 . 0 0 9 9}, 109.0306,107.0153$ & Delphinidin & $\mathrm{S}, \mathrm{L}$ \\
\hline 51 & 4.97 & $\mathrm{C}_{15} \mathrm{H}_{10} \mathrm{O}_{8}$ & 318.0368 & 318.0376 & -2.25 & $+\mathrm{HCOO}$ & $300.0266,264.0562,176.0132,148.0176$ & Quercetagetin & $\mathrm{L}$ \\
\hline 52 & 5.12 & $\mathrm{C}_{27} \mathrm{H}_{30} \mathrm{O}_{15}$ & 594.1609 & 594.1585 & 4.02 & $-\mathrm{H}$ & 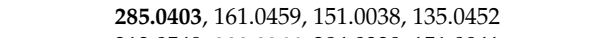 & Kaempferol-3-O-neohesperidoside & $\mathrm{R}$ \\
\hline 53 & 5.14 & $\mathrm{C}_{21} \mathrm{H}_{20} \mathrm{O}_{12}$ & 464.0945 & 464.0955 & -2.16 & $-\mathrm{H}$ & $313.0549, \mathbf{3 0 0 . 0 2 6 6}, 284.0330,151.0041$ & Quercimeritrin & $\mathrm{S}, \mathrm{L}, \mathrm{D}$ \\
\hline 54 & 5.17 & $\mathrm{C}_{21} \mathrm{H}_{22} \mathrm{O}_{11}$ & 450.1178 & 450.1162 & 3.61 & $-\mathrm{H}$ & 193.0156, 179.0574, 175.0051, 151.0052, 135.0468 & Dihydrokaempferol-5-O- $\beta$-D-glucopyranoside & $\mathrm{D}$ \\
\hline 55 & 5.24 & $\mathrm{C}_{15} \mathrm{H}_{10} \mathrm{O}_{6}$ & 286.0463 & 286.0477 & -4.95 & $-\mathrm{H}$ & $256.0372,177.0180,164.0487, \mathbf{1 5 0 . 0 3 0 0 ,} 123.0439,107.0134$ & $\omega$-Hydroxyemodin ${ }^{a}$ & $\mathrm{D}$ \\
\hline 56 & 5.25 & $\mathrm{C}_{17} \mathrm{H}_{16} \mathrm{O}_{9}$ & 364.0780 & 364.0794 & -3.53 & $+\mathrm{HCOO}$ & $337.0566,278.0432,202.0248,185.0254,149.0251$ & Bergaptol-O- $\beta$-D-glucopyranoside & $\mathrm{L}$ \\
\hline 57 & 5.26 & $\mathrm{C}_{15} \mathrm{H}_{12} \mathrm{O}_{7}$ & 304.0568 & 304.0583 & -4.92 & $-\mathrm{H}$ & $285.0366,243.0329,152.0099, \mathbf{1 5 0 . 0 3 0 0}, 125.0238$ & Dihydroquercetin & $\mathrm{D}$ \\
\hline 58 & 5.27 & $\mathrm{C}_{21} \mathrm{H}_{20} \mathrm{O}_{11}$ & 448.1005 & 448.1006 & -0.19 & $-\mathrm{H}$ & 285.0406, $283.0256,179.0569$ & Luteolin-7-O-glucopyranoside & $\mathrm{R}, \mathrm{D}$ \\
\hline 59 & 5.40 & $\mathrm{C}_{15} \mathrm{H}_{10} \mathrm{O}_{6}$ & 286.0479 & 286.0477 & 0.70 & $+\mathrm{H}$ & $\mathbf{1 4 9 . 0 2 1 6 , 1 3 9 . 0 3 7 1 , 1 2 3 . 0 4 3 3 , 1 1 1 . 0 4 3 9}$ & 7-Hydroxy-1-methoxy-2-methoxyxanthone ${ }^{a}$ & $\mathrm{~S}, \mathrm{~L}$ \\
\hline 60 & 5.57 & $\mathrm{C}_{41} \mathrm{H}_{32} \mathrm{O}_{26}$ & 940.1163 & 940.1182 & -1.96 & $-\mathrm{H}$ & 769.0887, 617.0782, 313.0565, 291.0150, 169.0158 & $1,2,3,4,6$-Penta-O-galloyl- $\beta$-D-glucopyranoside ${ }^{a}$ & $\mathrm{~S}, \mathrm{~L}$ \\
\hline 61 & 5.72 & $\mathrm{C}_{20} \mathrm{H}_{18} \mathrm{O}_{11}$ & 434.0853 & 434.0849 & 0.80 & $-\mathrm{H}$ & 300.0301, 195.0321, 151.0050, 109.0305 & Quercetin-3-O-arabinoside & $\mathrm{S}$ \\
\hline 62 & 5.76 & $\mathrm{C}_{30} \mathrm{H}_{36} \mathrm{O}_{8}$ & 524.2409 & 524.2410 & -0.19 & $+\mathrm{HCOO}$ & $453.1908,339.1256,195.0667,165.0570$ & Saucerneol $C^{a}$ & $\mathrm{R}$ \\
\hline 63 & 5.79 & $\mathrm{C}_{23} \mathrm{H}_{24} \mathrm{O}_{13}$ & 508.1224 & 508.1217 & 1.36 & $-\mathrm{H}$ & 315.0519, 207.0291, 193.0506, 151.0044, 137.0246 & Limocitrin-3-O- $\beta$-D-glucopyranoside ${ }^{a}$ & $\mathrm{~L}$ \\
\hline 64 & 5.83 & $\mathrm{C}_{27} \mathrm{H}_{30} \mathrm{O}_{14}$ & 578.1637 & 578.1636 & 0.24 & $-\mathrm{H}$ & 269.0475, 227.0364, 177.0203, $151.0050,119.0513$ & Apigenin-7-O- $\beta$-D-rutinoside & $\mathrm{D}$ \\
\hline 65 & 5.84 & $\mathrm{C}_{21} \mathrm{H}_{20} \mathrm{O}_{11}$ & 448.1016 & 448.1006 & 2.36 & $-\mathrm{H}$ & 295.0843, 284.0340, 179.0362, 151.0411, 123.0102 & Quercetin-3-O- $\alpha$-L-rhamnoside & $\mathrm{s}$ \\
\hline 66 & 5.86 & $\mathrm{C}_{14} \mathrm{H}_{18} \mathrm{O}_{3}$ & 234.1243 & 234.1256 & -4.58 & $+\mathrm{H}$ & $175.0746, \mathbf{1 6 3 . 0 7 4 6}, 133.0647,119.0860,111.0811$ & Lobetyol & $\mathrm{R}$ \\
\hline 67 & 6.02 & $\mathrm{C}_{26} \mathrm{H}_{38} \mathrm{O}_{13}$ & 558.2326 & 558.2312 & 2.37 & $+\mathrm{Na}$ & 217.1197, 199.1096, 145.0642, 128.0613, 115.0541 & Lobetyolinin & $\mathrm{R}$ \\
\hline 68 & 6.12 & $\mathrm{C}_{21} \mathrm{H}_{20} \mathrm{O}_{10}$ & 432.1040 & 432.1056 & -3.82 & $-\mathrm{H}$ & $268.0367,227.0341,177.0181,151.0037,124.0168$ & Cosmosiin & $\mathrm{D}$ \\
\hline 69 & 6.17 & $\mathrm{C}_{15} \mathrm{H}_{12} \mathrm{O}_{6}$ & 288.0643 & 288.0634 & 3.23 & $-\mathrm{H}$ & 271.0623, 177.0181, 151.0037, 133.0297, 125.0254, 107.0143 & Dihydrokaempferol & $\mathrm{D}$ \\
\hline 70 & 6.32 & $\mathrm{C}_{9} \mathrm{H}_{10} \mathrm{O}_{4}$ & 182.0584 & 182.0579 & 2.65 & $-\mathrm{H}$ & $166.0263,151.0040, \mathbf{1 3 5 . 0 4 5 2}, 108.0226$ & 2,6-Dimethoxy benzoic acid & $\mathrm{D}$ \\
\hline 71 & 6.59 & $\mathrm{C}_{21} \mathrm{H}_{24} \mathrm{O}_{7}$ & 388.1509 & 388.1522 & -2.96 & $+\mathrm{HCOO}$ & $358.1066,301.0369,243.0306,231.0308,151.0047$ & $\beta$-Hydroxyisovalerylshikonin ${ }^{a}$ & $\mathrm{D}$ \\
\hline 72 & 6.61 & $\mathrm{C}_{20} \mathrm{H}_{18} \mathrm{O}_{10}$ & 418.0892 & 418.0900 & -1.74 & $+\mathrm{HCOO}$ & 358.1066, 243.0306, 231.0308, 178.9997, 151.0047, 121.0304 & Cimicifugic acid $\mathrm{D}^{\mathrm{a}}$ & $\mathrm{D}$ \\
\hline 73 & 6.70 & $\mathrm{C}_{21} \mathrm{H}_{24} \mathrm{O}_{10}$ & 436.1373 & 436.1369 & 0.76 & $-\mathrm{H}$ & 273.0781, 255.0666, 179.0358, 149.0248, 123.0457 & Epiafzelechin-3-O- $\beta$-D-allopyranoside ${ }^{a}$ & $\mathrm{D}$ \\
\hline 74 & 6.75 & $\mathrm{C}_{42} \mathrm{H}_{68} \mathrm{O}_{16}$ & 828.4491 & 828.4507 & -1.99 & $+\mathrm{H}$ & 667.4052, 651.4104, 505.3529, 487.3428, 469.3321, 421.3113 & Platycosaponin A & $\mathrm{R}$ \\
\hline 75 & 6.79 & $\mathrm{C}_{22} \mathrm{H}_{22} \mathrm{O}_{10}$ & 446.1231 & 446.1213 & 3.66 & $+\mathrm{HCOO}$ & 285.0424, 187.0053, 163.0414, 124.0179 & Rhamnocitrin-3-O-rhamnoside a & $\mathrm{s}$ \\
\hline 76 & 6.81 & $\mathrm{C}_{20} \mathrm{H}_{28} \mathrm{O}_{8}$ & 396.1793 & 396.1784 & 2.03 & $+\mathrm{HCOO}$ & $215.1094,185.0984,159.0826,143.0724,125.0616$ & Lobetyolin & $\mathrm{R}$ \\
\hline $77^{*}$ & 6.85 & $\mathrm{C}_{64} \mathrm{H}_{104} \mathrm{O}_{34}$ & 1416.6388 & 1416.6409 & -1.49 & $+\mathrm{H}$ & 811.4487, 763.42581, 647.37911, 485.3261 & Deapio platycoside E & $\mathrm{R}$ \\
\hline 78 & 6.93 & $\mathrm{C}_{35} \mathrm{H}_{58} \mathrm{O}_{6}$ & 574.4227 & 574.4233 & -1.03 & $+\mathrm{H}$ & $472.3166,463.3096,378.2044,302.1716$ & $\alpha$-Spinasterol glucoside & $\mathrm{R}$ \\
\hline $79 *$ & 6.98 & $\mathrm{C}_{69} \mathrm{H}_{112} \mathrm{O}_{38}$ & 1548.6799 & 1548.6832 & -2.13 & $+\mathrm{H}$ & $1007.5104,845.4571,683.4034,521.3493,485.3282$ & Platycoside E & $\mathrm{R}$ \\
\hline 80 & 6.99 & $\mathrm{C}_{23} \mathrm{H}_{24} \mathrm{O}_{11}$ & 476.1314 & 476.1319 & -0.84 & $+\mathrm{HCOO}$ & $433.1097, \mathbf{3 4 5 . 0 8 1 9 ,} 313.0554,183.0309,151.0041$ & 5 -Hydroxy-6,4'-dimethoxy-flavone-7-O- $\beta$-D-gluco-pyranoside & $\mathrm{s}$ \\
\hline 81 & 7.35 & $\mathrm{C}_{29} \mathrm{H}_{46} \mathrm{O}_{4}$ & 458.3396 & 458.3396 & -0.05 & $+\mathrm{H}$ & $341.2455,217.1953,149.1333,121.1027$ & Neotigogenin acetate & $\mathrm{R}$ \\
\hline 82 & 7.57 & $\mathrm{C}_{58} \mathrm{H}_{94} \mathrm{O}_{29}$ & 1254.5905 & 1254.5881 & 1.95 & $+\mathrm{H}$ & $931.4894,845.4518,799.4485,295.1007$ & Deapioplatycodin $\mathrm{D}_{3}$ & $\mathrm{R}$ \\
\hline 83 * & 7.68 & $\mathrm{C}_{63} \mathrm{H}_{102} \mathrm{O}_{33}$ & 1386.6326 & 1386.6303 & 1.65 & $+\mathrm{H}$ & $\begin{array}{c}1255.5937,931.4894,845.4518,799.4484,665.3879, \\
441.1585\end{array}$ & Platycodin $\mathrm{D}_{3}$ & $\mathrm{R}$ \\
\hline 84 & 7.69 & $\mathrm{C}_{15} \mathrm{H}_{12} \mathrm{O}_{6}$ & 288.0629 & 288.0634 & -1.59 & $+\mathrm{H}$ & $255.0652,179.0353,163.0400, \mathbf{1 5 3 . 0 1 9 6}, 145.0295$ & 3-Hydroxynaringenin ${ }^{a}$ & $\mathrm{D}$ \\
\hline $85^{*}$ & 7.77 & $\mathrm{C}_{15} \mathrm{H}_{10} \mathrm{O}_{7}$ & 302.0422 & 302.0427 & -1.40 & $+\mathrm{H}$ & $243.0319,151.0055,125.0260,107.0157$ & Quercetin & $\mathrm{S}, \mathrm{L}$ \\
\hline 86 & 7.86 & $\mathrm{C}_{15} \mathrm{H}_{10} \mathrm{O}_{6}$ & 286.0488 & 286.0477 & 3.61 & $+\mathrm{H}$ & $269.0460,257.0450, \mathbf{2 4 1 . 0 4 9 0 ,} 161.0239,135.0453$ & 6-Hydroxyaloeemodin ${ }^{a}$ & $\mathrm{D}$ \\
\hline 87 & 7.91 & $\mathrm{C}_{30} \mathrm{H}_{26} \mathrm{O}_{13}$ & 594.1373 & 594.1373 & -0.14 & $-\mathrm{H}$ & $447.0966,429.0832,285.0440,145.0316,119.0513$ & Buddlenoid $\mathrm{A}^{\mathrm{a}}$ & $\mathrm{S}, \mathrm{L}$ \\
\hline 88 & 7.92 & $\mathrm{C}_{47} \mathrm{H}_{76} \mathrm{O}_{20}$ & 960.4934 & 960.4930 & 0.39 & $+\mathrm{HCOO}$ & $869.4537,715.3371,529.2698,295.2034$ & Platycoside F & $\mathrm{R}$ \\
\hline 89 * & 7.94 & $\mathrm{C}_{63} \mathrm{H}_{102} \mathrm{O}_{32}$ & 1370.6373 & 1370.6354 & 1.40 & $+\mathrm{H}$ & 827.4398, 783.4476, 637.3944, 459.3430, 409.3090, $\mathbf{3 2 5 . 1 1 3 0}$ & Platycoside $\mathrm{G}_{3}$ & $\mathrm{R}$ \\
\hline 90 & 8.33 & $\mathrm{C}_{57} \mathrm{H}_{90} \mathrm{O}_{29}$ & 1238.5577 & 1238.5568 & 0.71 & $+\mathrm{H}$ & $\begin{array}{c}1107.5237,957.4692,895.4676,811.4125,697.3760, \\
661.3582,485.3245,409.3094\end{array}$ & Platyconic acid A & $\mathrm{D}$ \\
\hline
\end{tabular}


Table 1. Cont

\begin{tabular}{|c|c|c|c|c|c|c|c|c|c|}
\hline No. & $\begin{array}{c}t_{\mathrm{R}} \\
(\mathrm{min})\end{array}$ & Formula & $\begin{array}{l}\text { Experimental } \\
\text { (Da) }\end{array}$ & $\begin{array}{l}\text { Theoretical } \\
\text { (Da) }\end{array}$ & $\begin{array}{c}\text { Mass Error } \\
(\mathrm{ppm})\end{array}$ & Adducts & $\mathrm{MS}^{\mathrm{E}}$ Fragmentation & Component Name & Source \\
\hline $91 *$ & 8.46 & $\mathrm{C}_{52} \mathrm{H}_{84} \mathrm{O}_{24}$ & 1092.5397 & 1092.5353 & 4.07 & $-\mathrm{H}$ & $\begin{array}{c}959.4846,941.4753,681.3871,663.3768,649.3607,503.3364 \\
485.3366,295.1038,277.0942\end{array}$ & Deapioplatycodin D & $\mathrm{R}$ \\
\hline 92 & 8.48 & $\mathrm{C}_{59} \mathrm{H}_{92} \mathrm{O}_{30}$ & 1280.5649 & 1280.5673 & -1.90 & $+\mathrm{H}$ & $\begin{array}{c}1017.4875,999.4760,931.4860,829.4192,697.3796 \\
679.3651,651.3761,519.3316,503.3334,487.3377\end{array}$ & Platycodin L & $\mathrm{R}$ \\
\hline $93 *$ & 8.51 & $\mathrm{C}_{58} \mathrm{H}_{94} \mathrm{O}_{29}$ & 1254.5847 & 1254.5881 & -2.65 & $+\mathrm{H}$ & $\begin{array}{c}931.4894,845.4518,799.4485,483.3065,457.1533,427.1433 \\
\text { 325.1116, } 295.1007\end{array}$ & Deapioplatycodin $\mathrm{D}_{2}$ & $\mathrm{R}$ \\
\hline $94 *$ & 8.62 & $\mathrm{C}_{63} \mathrm{H}_{102} \mathrm{O}_{33}$ & 1386.6300 & 1386.6303 & -0.26 & $+\mathrm{H}$ & $\begin{array}{c}977.4981,845.4558,829.4604,683.4031,667.4073,653.3919 \\
\mathbf{5 2 1 . 3 4 8 8 , 4 8 5 . 3 2 7 3}\end{array}$ & Platycodin $\mathrm{D}_{2}$ & $\mathrm{R}$ \\
\hline $95^{*}$ & 8.68 & $\mathrm{C}_{57} \mathrm{H}_{92} \mathrm{O}_{28}$ & 1224.5778 & 1224.5775 & 0.23 & $+\mathrm{H}$ & 799.4485, 683.3961, 667.4052, 521.3444, 503.3364, 485.3257 & Platycodin D & $\mathrm{R}, \mathrm{D}$ \\
\hline $96^{*}$ & 8.73 & $\mathrm{C}_{65} \mathrm{H}_{104} \mathrm{O}_{34}$ & 1428.6407 & 1428.6409 & -0.15 & $+\mathrm{H}$ & $\begin{array}{c}1297.6065,1165.5621,845.4520,841.4580,681.3837 \\
665.3903, \mathbf{6 5 3 . 3 8 8 4 ,} 617.3663,519.3298,485.3243\end{array}$ & 2'-O-Acetylplatycodin $\mathrm{D}_{2}$ & $\mathrm{R}, \mathrm{D}$ \\
\hline 97 & 8.78 & $\mathrm{C}_{59} \mathrm{H}_{94} \mathrm{O}_{29}$ & 1266.5869 & 1266.5881 & -0.93 & $+\mathrm{H}$ & $\begin{array}{c}1003.5108,841.4569,823.4458,683.3979,189.0749 \\
171.0641\end{array}$ & Platycodin A & $\mathrm{R}, \mathrm{D}$ \\
\hline 98 & 8.80 & $\mathrm{C}_{65} \mathrm{H}_{104} \mathrm{O}_{33}$ & 1412.6458 & 1412.6460 & -0.16 & $+\mathrm{H}$ & $\begin{array}{c}985.4990, \\
823.4461,635.3794,617.3695,503.3369,453.1605, \\
321.1182,303.1076,189.5707\end{array}$ & $3^{\prime \prime}$-O-Acetylpolygalacin $\mathrm{D}_{2}$ & $\mathrm{R}$ \\
\hline 99 & 8.86 & $\mathrm{C}_{15} \mathrm{H}_{10} \mathrm{O}_{5}$ & 270.0539 & 270.0528 & 4.15 & $-\mathrm{H}$ & $\mathbf{1 5 1 . 0 0 4 3 ,}, 123.0099,117.0359,107.0154$ & Apigenol & $\mathrm{D}$ \\
\hline 100 & 8.87 & $\mathrm{C}_{52} \mathrm{H}_{82} \mathrm{O}_{25}$ & 1106.5163 & 1106.5145 & 1.57 & $+\mathrm{H}$ & $\begin{array}{c}975.4806,931.4908,829.4243,811.4113,697.3814,679.3695 \\
517.3151,503.3373,455.3161\end{array}$ & Platyconic acid C & $\mathrm{R}$ \\
\hline 101 & 8.94 & $\mathrm{C}_{59} \mathrm{H}_{92} \mathrm{O}_{30}$ & 1280.5705 & 1280.5673 & 2.47 & $+\mathrm{H}$ & $\begin{array}{c}1017.4875,829.4192,697.3796,637.3939,519.3316 \\
321.1178\end{array}$ & Platycodin K & $\mathrm{R}, \mathrm{D}$ \\
\hline 102 & 9.04 & $\mathrm{C}_{54} \mathrm{H}_{86} \mathrm{O}_{25}$ & 1134.5444 & 1134.5458 & -1.23 & $+\mathrm{H}$ & $\begin{array}{c}1003.5108,841.4569,823.4458, \text { 683.3979, 321.1160, } \\
189.0749\end{array}$ & Platycoside B & $\mathrm{R}$ \\
\hline $103 *$ & 9.10 & $\mathrm{C}_{65} \mathrm{H}_{104} \mathrm{O}_{34}$ & 1428.6370 & 1428.6409 & -2.71 & $+\mathrm{H}$ & $\begin{array}{c}\text { 1297.6065, 955.4894, 841.4580, 813.4279, } 797.4332 \\
681.3837,665.3903,653.3884,635.3780\end{array}$ & 3'-O-acetyl-platycodin $\mathrm{D}_{2}$ & $\mathrm{R}$ \\
\hline 104 & 9.11 & $\mathrm{C}_{15} \mathrm{H}_{10} \mathrm{O}_{6}$ & 286.0483 & 286.0477 & 1.85 & $+\mathrm{H}$ & 231.0662, 229.0504, 195.0289, 153.0187, & Kaempferol & $\mathrm{L}$ \\
\hline 105 & 9.14 & $\mathrm{C}_{20} \mathrm{H}_{24} \mathrm{O}_{11}$ & 440.1314 & 440.1319 & -1.01 & $-\mathrm{H}$ & $393.0860,303.0523,257.0104,231.0303,177.0204$ & $(-)$-Chebulic acid triethyl ester ${ }^{a}$ & $\mathrm{~S}, \mathrm{~L}$ \\
\hline 106 & 9.18 & $\mathrm{C}_{65} \mathrm{H}_{104} \mathrm{O}_{33}$ & 1412.6430 & 1412.6460 & -2.14 & $+\mathrm{H}$ & 823.4461, 503.3369, 485.3255, 455.3156, 321.1182, 189.0757 & $2^{\prime \prime}-\mathrm{O}$-acetylpolygalacin $\mathrm{D}_{2}$ & $\mathrm{R}, \mathrm{D}$ \\
\hline 107 & 9.23 & $\mathrm{C}_{59} \mathrm{H}_{94} \mathrm{O}_{28}$ & 1250.5904 & 1250.5932 & -2.23 & $-\mathrm{H}$ & 1208.5857, 1159.5571, 635.3812, 499.3046, 131.0337 & $2^{\prime}-\mathrm{O}$-acetyl Polygalacin D & $\mathrm{R}$ \\
\hline 108 & 9.32 & $\mathrm{C}_{20} \mathrm{H}_{22} \mathrm{O}_{11}$ & 438.1170 & 438.1162 & 1.78 & $-\mathrm{H}$ & 419.0956, 235.0654, 163.0050 & $6^{\prime}-\mathrm{O}$-Galloyl-homoarbutin a & $\mathrm{S}, \mathrm{L}$ \\
\hline 109 & 9.37 & $\mathrm{C}_{54} \mathrm{H}_{84} \mathrm{O}_{26}$ & 1148.5293 & 1148.5251 & 3.63 & $+\mathrm{H}$ & $\begin{array}{c}\text { 1017.4908, 999.4786, 535.3279, 631.3477, 517.3170, } \\
499.3050,453.3001,321.1190,189.0764\end{array}$ & Platyconic acid D & $\mathrm{R}$ \\
\hline 110 & 9.45 & $\mathrm{C}_{35} \mathrm{H}_{54} \mathrm{O}_{11}$ & 650.3666 & 650.3666 & 0.04 & $+\mathrm{HCOO}$ & $451.2830,441.2997,197.1183, \mathbf{1 4 9 . 0 4 6 5 ,} 131.0354$ & $15 \alpha$-Hydroxy-ximicifugoside $\mathrm{H}_{2}$ a & $\mathrm{R}$ \\
\hline 111 & 9.59 & $\mathrm{C}_{37} \mathrm{H}_{60} \mathrm{O}_{12}$ & 696.4087 & 696.4085 & 0.28 & $-\mathrm{H}$ & $487.3424,469.3302,425.3438$ & 3-O-D-glucopyranosyl platycodigenin methyl ester & $\mathrm{s}$ \\
\hline 112 & 9.80 & $\mathrm{C}_{30} \mathrm{H}_{42} \mathrm{O}_{7}$ & 514.2938 & 514.2931 & 1.41 & $-\mathrm{H}$ & 436.2610, 319.1910, 301.1814, 265.1468 & Marstenacigenin A & $\mathrm{R}$ \\
\hline 113 & 9.91 & $\mathrm{C}_{36} \mathrm{H}_{58} \mathrm{O}_{12}$ & 682.3893 & 682.3928 & -4.81 & $+\mathrm{HCOO}$ & $635.3797,449.3263,407.2948,179.0565$ & 3-O-D-glucopyranosyl platycodigenin & $\mathrm{R}$ \\
\hline 114 & 9.94 & $\mathrm{C}_{19} \mathrm{H}_{16} \mathrm{O}_{7}$ & 356.0886 & 356.0896 & -2.55 & $+\mathrm{HCOO}$ & $401.0868,313.0718, \mathbf{1 2 1 . 0 2 9 7}$ & 6-Formyl-isoophiopogonanone $\mathrm{A}^{\mathrm{a}}$ & $\mathrm{R}$ \\
\hline 115 & 10.17 & $\mathrm{C}_{15} \mathrm{H}_{18} \mathrm{O}_{3}$ & 246.1258 & 246.1256 & 0.84 & $+\mathrm{H}$ & 229.1220, $163.0756,149.0598,119.0865,105.0713$ & Curcolone ${ }^{a}$ & $\mathrm{~S}, \mathrm{~L}$ \\
\hline 116 & 10.25 & $\mathrm{C}_{18} \mathrm{H}_{34} \mathrm{O}_{5}$ & 330.2418 & 330.2406 & 3.57 & $-\mathrm{H}$ & 311.2224, 293.2140, 211.1348, 185.1189, 129.0928 & Sanleng acid a & $\mathrm{R}, \mathrm{S}, \mathrm{D}$ \\
\hline 117 & 10.91 & $\mathrm{C}_{15} \mathrm{H}_{14} \mathrm{O}_{4}$ & 258.0901 & 258.0892 & 3.45 & $-\mathrm{H}$ & $239.0705,163.0397, \mathbf{1 5 1 . 0 4 2 1}, 133.0313,121.0296$ & Benzyl-2-hydroxy-6-methoxybenzoate & $\mathrm{D}$ \\
\hline $118^{*}$ & 10.95 & $\mathrm{C}_{15} \mathrm{H}_{20} \mathrm{O}_{3}$ & 248.1413 & 248.1412 & 0.27 & $+\mathrm{H}$ & $231.1379,219.1381,203.1425,119.0864,107.0867$ & Atractylenolide III & $\mathrm{L}$ \\
\hline 119 & 11.13 & $\mathrm{C}_{15} \mathrm{H}_{20} \mathrm{O}_{2}$ & 232.1464 & 232.1463 & 0.24 & $+\mathrm{H}$ & 215.1424, 187.1486, 159.1172, 135.1174, 107.0867 & Atractylenolide II & $\mathrm{S}, \mathrm{L}$ \\
\hline 120 & 12.19 & $\mathrm{C}_{16} \mathrm{H}_{12} \mathrm{O}_{6}$ & 300.0637 & 300.0634 & 1.18 & $+\mathrm{H}$ & 285.0761, 242.0571, $167.0340,136.0162,108.0215$ & 5-Methyl kaempferol & $\mathrm{S}, \mathrm{L}$ \\
\hline 121 & 12.26 & $\mathrm{C}_{17} \mathrm{H}_{14} \mathrm{O}_{6}$ & 314.0794 & 314.0790 & 1.05 & $+\mathrm{H}$ & $299.0552,275.0673,257.0445,161.0597,139.0397$ & 3,, 5 -Dihydroxy-7, $4^{\prime}$-dimethoxy flavone & $\mathrm{s}$ \\
\hline 122 & 12.94 & $\mathrm{C}_{17} \mathrm{H}_{26} \mathrm{O}_{4}$ & 294.1833 & 294.1831 & 0.56 & $-\mathrm{H}$ & $235.1341,141.0919, \mathbf{1 2 9 . 0 9 2 4}$ & 6-Gingerol ${ }^{\text {a }}$ & $\mathrm{R}$ \\
\hline 123 & 13.46 & $\mathrm{C}_{36} \mathrm{H}_{58} \mathrm{O}_{12}$ & 682.3905 & 682.3928 & -3.36 & $-\mathrm{H}$ & $635.3787,473.3258,443.3119,425.3020,179.0553$ & Trachelosperoside B-1 ${ }^{\text {a }}$ & $\mathrm{D}$ \\
\hline 124 & 13.68 & $\mathrm{C}_{30} \mathrm{H}_{48} \mathrm{O}_{5}$ & 488.3514 & 488.3502 & 2.47 & $-\mathrm{H}$ & $455.3548,439.3599, \mathbf{2 8 1 . 2 5 0 3}, 293.2127,171.1035$ & $2 \alpha, 19 \alpha$-Dihydroxyursolic acid & $\mathrm{L}$ \\
\hline 125 & 13.91 & $\mathrm{C}_{18} \mathrm{H}_{16} \mathrm{O}_{6}$ & 328.0949 & 328.0947 & 0.72 & $+\mathrm{H}$ & $314.0777,296.0677,184.0737,136.0166$ & $4^{\prime}, 7$-Dimethyltectorigenin ${ }^{a}$ & $\mathrm{~S}, \mathrm{~L}$ \\
\hline
\end{tabular}


Table 1. Cont

\begin{tabular}{|c|c|c|c|c|c|c|c|c|c|}
\hline No. & $\begin{array}{c}t_{\mathrm{R}} \\
(\mathrm{min})\end{array}$ & Formula & $\begin{array}{c}\text { Experimental } \\
\text { (Da) }\end{array}$ & $\begin{array}{l}\text { Theoretical } \\
\text { (Da) }\end{array}$ & $\begin{array}{l}\text { Mass Error } \\
(\mathrm{ppm})\end{array}$ & Adducts & $\mathrm{MS}^{\mathrm{E}}$ Fragmentation & Component Name & Source \\
\hline $126^{*}$ & 14.58 & $\mathrm{C} 18 \mathrm{H} 34 \mathrm{O} 4$ & 314.2466 & 314.2457 & 2.86 & $-\mathrm{H}$ & 201.1140, 199.0980, 155.1082, 127.1135 & Dibutyl sebacate & $\mathrm{R}$ \\
\hline 127 & 14.85 & $\mathrm{C}_{19} \mathrm{H}_{18} \mathrm{O}_{7}$ & 358.1051 & 358.1053 & -0.47 & $+\mathrm{H}$ & $343.0809,326.0778,301.0705,283.0599$ & $\begin{array}{l}\text { 3,4-Dihydro-6,8-dihydroxyl-3-(2'-acetyl-3'-hydroxyl-5'- } \\
\text { methoxyphenyl)methyl-1H-[2] benzoplyran-1-one a }\end{array}$ & $\mathrm{S}, \mathrm{L}$ \\
\hline 128 & 14.86 & $\mathrm{C}_{17} \mathrm{H}_{30} \mathrm{O}_{2}$ & 266.2258 & 266.2246 & 3.76 & $+\mathrm{HCOO}$ & 311.2240, 155.1083, 139.1137 & $\begin{array}{l}\text { Methyl } 7,10 \text {-hexadecadienoate } \\
\text { Mentive }\end{array}$ & $\mathrm{R}$ \\
\hline 129 & 15.36 & $\mathrm{C}_{30} \mathrm{H}_{48} \mathrm{O}_{7}$ & 520.3385 & 520.3400 & -2.93 & $-\mathrm{H}$ & $476.2774,473.3256,443.3168,425.3093,407.2940,395.2941$ & Platycodigenin & $\mathrm{D}$ \\
\hline 130 & 15.39 & $\mathrm{C}_{17} \mathrm{H}_{14} \mathrm{O}_{5}$ & 298.0843 & 298.0841 & 0.51 & $+\mathrm{H}$ & $284.0679,256.0730,241.0495,167.0339,133.0648$ & 5-Hydroxy-7, $4^{\prime}$-dimethoxyflavanone & $\mathrm{S}, \mathrm{L}$ \\
\hline 131 & 15.57 & $\mathrm{C}_{26} \mathrm{H}_{40} \mathrm{O}_{6}$ & 448.2818 & 448.2825 & -1.59 & $+\mathrm{H}$ & $393.2636,350.1875,242.1877$ & Tenasogenin ${ }^{\mathrm{a}}$ & $\mathrm{R}$ \\
\hline 132 & 15.89 & $\mathrm{C}_{14} \mathrm{H}_{20} \mathrm{O}$ & 204.1513 & 204.1514 & -0.51 & $+\mathrm{H}$ & $163.1118,159.1169,149.0956,119.0863, \mathbf{1 0 7 . 0 5 0 2}$ & 2-(p-Anisyl)-5-methyl-1-hexen & $\mathrm{L}$ \\
\hline 133 & 16.28 & $\mathrm{C}_{18} \mathrm{H}_{16} \mathrm{O}_{6}$ & 328.0957 & 328.0947 & 2.95 & $+\mathrm{H}$ & $314.0790,299.0550,286.0830,271.0604,150.0314$ & 5-Hydro-7, 8, 2'-trimethoxyflavanone & $\mathrm{S}, \mathrm{L}$ \\
\hline 134 & 16.57 & $\mathrm{C}_{32} \mathrm{H}_{44} \mathrm{O}_{9}$ & 572.2965 & 572.2985 & -3.51 & $-\mathrm{H}$ & 481.2572, $429.2997,227.0350,183.1043$ & Ganoderic acid $\mathrm{H}^{\text {a }}$ & $\mathrm{L}$ \\
\hline 135 & 17.23 & $\mathrm{C}_{30} \mathrm{H}_{48} \mathrm{O}_{4}$ & 472.3550 & 472.3553 & -0.49 & $-\mathrm{H}$ & $471.3448,437.3061,419.2937,339.2705,253.2187$ & $2 \alpha$-Hydroxybetulinic acid & $\mathrm{S}, \mathrm{L}$ \\
\hline 136 & 17.62 & $\mathrm{C}_{16} \mathrm{H}_{30} \mathrm{O}_{2}$ & 254.2252 & 254.2246 & 2.21 & $+\mathrm{Na}$ & 207.1743, 165.1274, 143.1067, 125.0961 & Palmitoleic acid & $\mathrm{R}$ \\
\hline 137 & 17.78 & $\mathrm{C}_{18} \mathrm{H}_{34} \mathrm{O}_{3}$ & 298.2505 & 298.2508 & -1.05 & $-\mathrm{H}$ & 217.1615, 195.1391, 183.1401, 113.0984 & Ricinoleic acid & $\mathrm{D}$ \\
\hline 138 & 18.00 & $\mathrm{C}_{18} \mathrm{H}_{30} \mathrm{O}_{3}$ & 294.2203 & 294.2195 & 2.51 & $+\mathrm{Na}$ & $277.2177,165.1284,151.1127,109.1035$ & $(E, E)$-9-Oxooctadeca-10,12-dienoic acid a & $\mathrm{R}$ \\
\hline 139 & 18.01 & $\mathrm{C}_{18} \mathrm{H}_{28} \mathrm{O}_{2}$ & 276.2100 & 276.2089 & 3.85 & $+\mathrm{H}$ & 179.1424, $135.1180,119.0862$ & Stearidonic acid & $\mathrm{R}$ \\
\hline 140 & 18.26 & $\mathrm{C}_{28} \mathrm{H}_{42} \mathrm{~N}_{4} \mathrm{O}_{6}$ & 530.3100 & 530.3104 & -0.77 & $-\mathrm{H}$ & $529.3027,511.2928,293.2163$ & Kukoamine $\mathrm{A}^{\mathrm{a}}$ & $\mathrm{R}$ \\
\hline 141 & 19.02 & $\mathrm{C}_{18} \mathrm{H}_{32} \mathrm{O}_{3}$ & 296.2358 & 296.2351 & 2.19 & $+\mathrm{Na}$ & $279.2312,161.1323, \mathbf{1 4 7 . 1 1 6 5}, 133.1018,121.1023$ & Coronaric acid & $\mathrm{R}$ \\
\hline 142 & 19.23 & $\mathrm{C}_{28} \mathrm{H}_{40} \mathrm{O}_{5}$ & 456.2878 & 456.2876 & 0.46 & $-\mathrm{H}$ & $409.2359,343.1925,339.2004,275.2022$ & Siraitic acid $\mathrm{D}^{\mathrm{a}}$ & $\mathrm{R}$ \\
\hline 143 & 20.35 & $\mathrm{C}_{32} \mathrm{H}_{50} \mathrm{O}_{5}$ & 514.3662 & 514.3658 & 0.81 & $-\mathrm{H}$ & $495.3495,469.3702,451.3596,449.3449$ & $19 \alpha$-Hydroxy-3-acetyl-ursolic acid & $\mathrm{s}$ \\
\hline 144 & 20.39 & $\mathrm{C}_{30} \mathrm{H}_{46} \mathrm{O}_{3}$ & 454.3452 & 454.3447 & 1.03 & $+\mathrm{H}$ & $437.3422,409.3470,247.1695,203.1796,189.1642$ & Oleanonic acid & $\mathrm{s}$ \\
\hline 145 & 20.77 & $\mathrm{C}_{30} \mathrm{H}_{48} \mathrm{O}_{3}$ & 456.3604 & 456.3603 & 0.13 & $-\mathrm{H}$ & $455.3531,443.3528,233.1561$ & 3-Epioleanolic acid & $\mathrm{s}$ \\
\hline 146 & 20.78 & $\mathrm{C}_{33} \mathrm{H}_{36} \mathrm{~N}_{4} \mathrm{O}_{6}$ & 584.2660 & 584.2635 & 4.08 & $+\mathrm{Na}$ & $567.2589,535.2340,501.2257,467.20432,417.1830$ & Bilirubin ${ }^{a}$ & $\mathrm{~L}$ \\
\hline 147 & 21.49 & $\mathrm{C}_{15} \mathrm{H}_{30} \mathrm{O}$ & 226.2309 & 226.2297 & 4.48 & $+\mathrm{HCOO}$ & 271.2302, 197.1911, 195.1754 & $\mathrm{n}$-Pentadecanal & $\mathrm{s}$ \\
\hline 148 & 22.20 & $\mathrm{C}_{30} \mathrm{H}_{50} \mathrm{O}_{2}$ & 442.3803 & 442.3811 & -1.76 & $+\mathrm{H}$ & 425.3776, 407.3666, 217.1950, 203.1791, 189.1641 & Betulin & $\mathrm{R}$ \\
\hline 149 * & 22.93 & $\mathrm{C}_{18} \mathrm{H}_{30} \mathrm{O}_{2}$ & 280.2402 & 280.2400 & -0.25 & $-\mathrm{H}$ & 149.0972 & Linolenic acid & $\mathrm{R}$ \\
\hline 150 * & 22.95 & $\mathrm{C}_{19} \mathrm{H}_{38} \mathrm{O}_{4}$ & 330.2774 & 330.2770 & 1.00 & $+\mathrm{Na}$ & $313.2738,239.2368$ & 1-Monopalmitin & $\mathrm{s}$ \\
\hline 151 & 22.98 & $\mathrm{C}_{16} \mathrm{H}_{32} \mathrm{O}$ & 240.2452 & 240.2453 & -0.47 & $+\mathrm{Na}$ & $263.2344,125.1317, \mathbf{1 1 1 . 1 1 7 5}$ & $n$-Hexadecanal & $\mathrm{D}$ \\
\hline 152 & 24.06 & $\mathrm{C}_{21} \mathrm{H}_{42} \mathrm{O}$ & 310.3240 & 310.3236 & 1.28 & $+\mathrm{HCOO}$ & $355.3214, \mathbf{1 2 5 . 0 9 7 2}$ & $n$-Henicosanal & $\mathrm{s}$ \\
\hline 153 & 24.40 & $\mathrm{C}_{16} \mathrm{H}_{32} \mathrm{O}_{2}$ & 256.2401 & 256.2402 & -0.49 & $-\mathrm{H}$ & 241.2176, 237.226, 227.2019, 125.0976 & Palmitic acid & $\mathrm{s}$ \\
\hline 154 & 24.74 & $\mathrm{C}_{18} \mathrm{H}_{34} \mathrm{O}_{2}$ & 282.2569 & 282.2559 & 3.70 & $-\mathrm{H}$ & $253.2185,163.1132, \mathbf{1 2 5 . 0 9 8 2 ,} 111.0825$ & Ethyl palmitate & $\mathrm{R}$ \\
\hline 155 & 25.73 & $\mathrm{C}_{29} \mathrm{H}_{46} \mathrm{O}$ & 410.3565 & 410.3549 & 4.03 & $+\mathrm{H}$ & $395.3680,203.1799,145.1021,133.1019$ & $\Delta 7$-stigmasterol & $\mathrm{R}$ \\
\hline 156 & 26.87 & $\mathrm{C}_{24} \mathrm{H}_{38} \mathrm{O}_{4}$ & 390.2771 & 390.2770 & 0.21 & $+\mathrm{H}$ & $301.1413,189.0156,165.0905, \mathbf{1 4 9 . 0 2 3 5}$ & Bis(2-ethylhexyl)phthalate & $\mathrm{R}$ \\
\hline 157 & 27.09 & $\mathrm{C}_{22} \mathrm{H}_{43} \mathrm{NO}$ & 337.3356 & 337.3345 & 3.47 & $+\mathrm{H}$ & $321.3149, \mathbf{2 1 2 . 2 0 1 4}, 198.1857,153.1275$ & Erucic amide ${ }^{\mathrm{a}}$ & $\mathrm{R}$ \\
\hline 158 & 27.63 & $\mathrm{C}_{20} \mathrm{H}_{40} \mathrm{O}$ & 296.3093 & 296.3079 & 4.10 & $+\mathrm{HCOO}$ & $251.2393, \mathbf{1 7 9 . 1 4 5 9 ,} 113.0987$ & Phytol & $\mathrm{s}$ \\
\hline 159 * & 28.49 & $\mathrm{C}_{29} \mathrm{H}_{48} \mathrm{O}$ & 412.3695 & 412.3705 & -2.48 & $+\mathrm{H}$ & $135.1178,109.1025$ & Stigmasterol & $\mathrm{R}$ \\
\hline
\end{tabular}

* Identified with a reference standard. ${ }^{a}$ Tentatively new identifications in Campanulaceae. The fragment ion mass highlighted as bold font is the characteristic MS fragmentation for each compound. 
Their identical MS fragment pattern were similar. But according to the literature, the C3-glucoside was eluted earlier than the C2-glucoside [26-28] in the ESI-BPI chromatogram, so the compound with the earlier RT was identified as the C3-glucoside, 3"-O-acetylpolygalacin D2, and the other one with the later RT was identified as the C2-glucoside, 2"-O-acetylpolygalacin D2.

\subsection{Biomarker Discovery for Differentiating Four Parts of PG}

The PCA 2D plots of the samples from the root, stem, leaf and seed groups were classified in four clusters according to their common spectral characteristics (Figure 3). That means the four parts of PG could be easily differentiated.
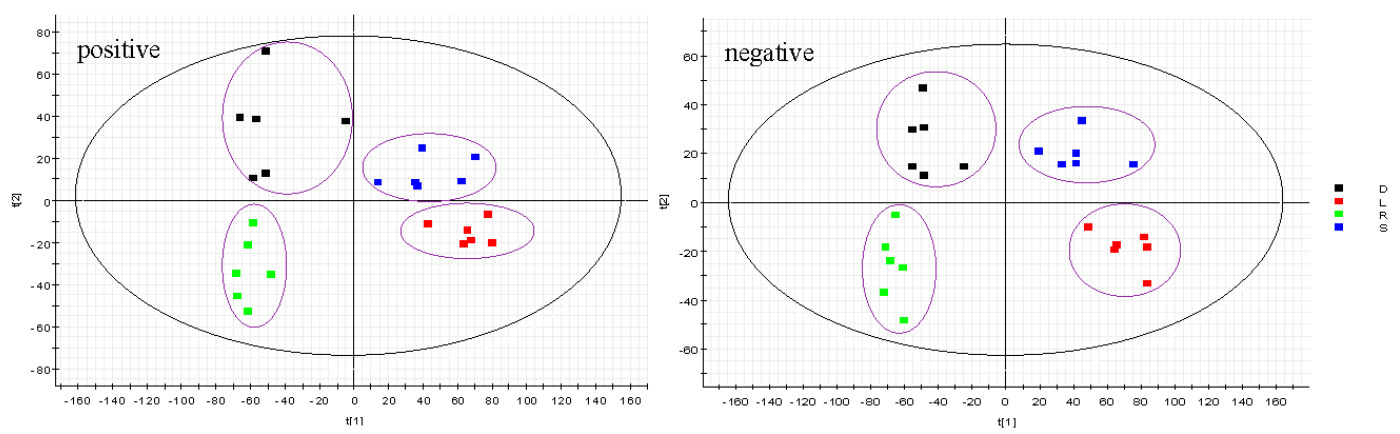

Figure 3. PCA of root (R), stem (S), leaf (L) and seed (D) of PG in positive mode and negative mode.

In order to differentiate one part from other three parts, the OPLS-DA models were built in both positive and negative modes. Then, OPLS-DA score plot, S-plot, variable trend and VIP (variable importance in the projection) values were obtained to understand which variables are the responsible for this sample separation [29]. Based on VIP values (VIP $>4$ ) (Figure 4) and $p$ values $(p<0.05)$ [30] from univariate statistical analysis, 38 robust known biomarkers enabling the differentiation among root, stem, leaf and seed, were discovered and marked in S-plots (Figure 5). In order to systematically evaluate the biomarkers, a heatmap was generated from these biomarkers (shown in Figure 6), which shows distinct segregation among the four parts.

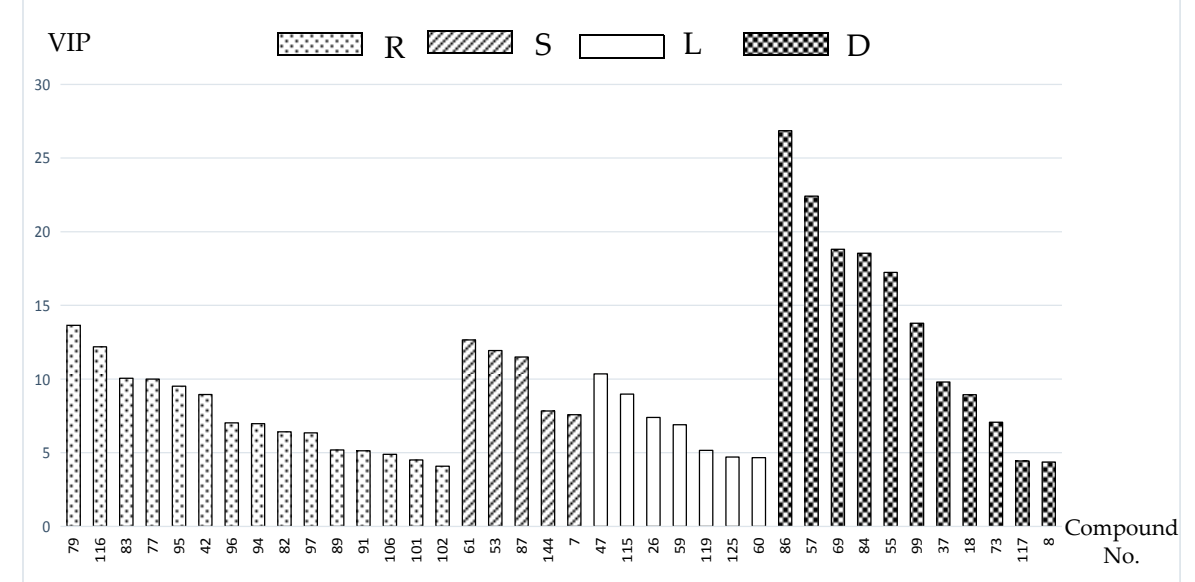

Figure 4. VIP value obtained from OPLS-DA model of the potential markers in root (R), stem (S), leaf (L) and seed (D) of PG. 

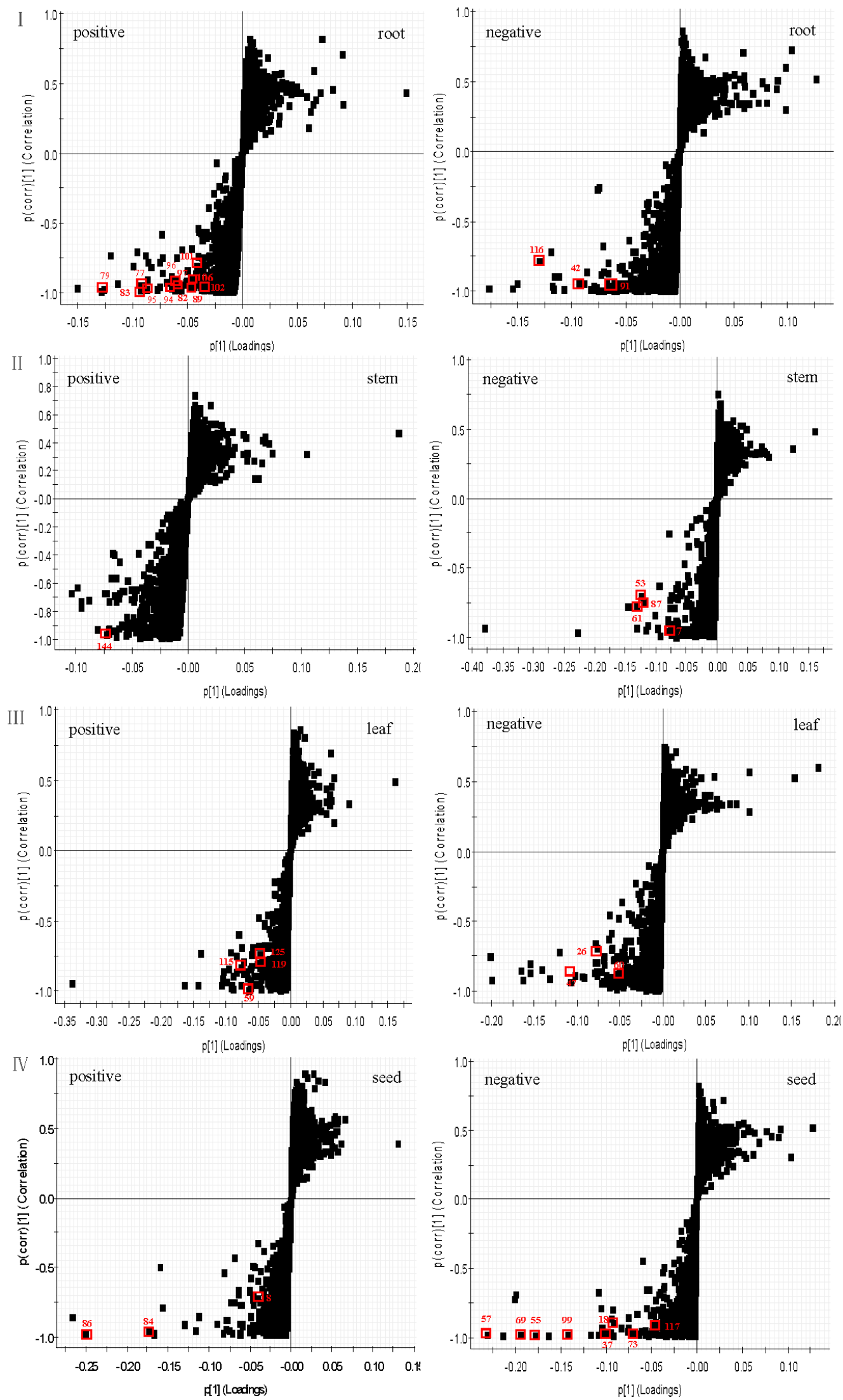

Figure 5. The OPLS-DA/S-plots of root (I), stem (II), leaf (III) and seed (IV) of PG in positive mode and negative mode. 


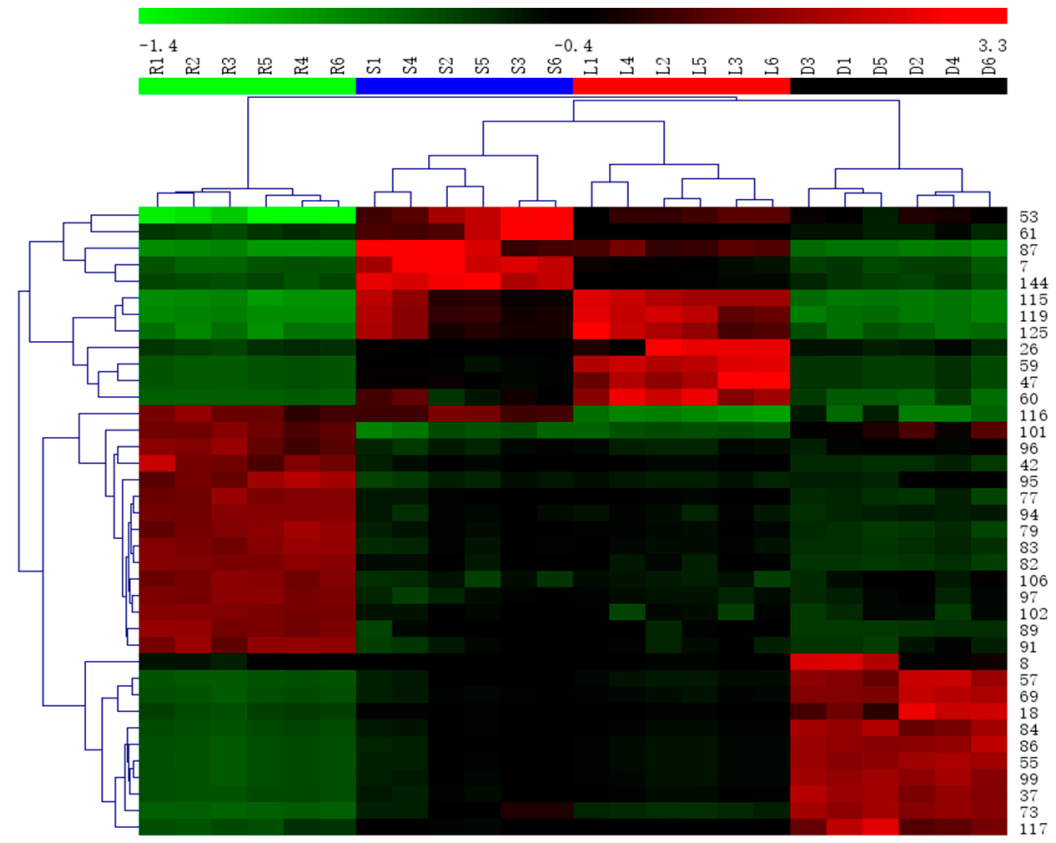

Figure 6. Heatmap visualizing the intensities of potential biomarkers.

\section{Discussion}

There are 73, 42, 35, 44 compounds that were characterized from the root, stem, leaf and seed, respectively. As the results show, 95 compounds were identified in ESI(-) mode and 64 compounds were identified in ESI(+) mode. According to the BPI chromatograms of the four parts of PG, it seems that $\operatorname{ESI}(-)$ ionization mode is better than $\mathrm{ESI}(+)$ based on the quantity and the responses of the identified compounds, but it is still necessary to run the ESI $(+)$ mode because some compounds showed better respond than in ESI(-) mode.

Compared with the results from previous studies [2,8,16,31,32], 56 chemical components were identified for the first time in Campanulaceae. The stem, leaf and seed contain more flavonoids but few saponins that can be easily discriminated from the root. In previous study, various metabolites in Korean Platycodon grandiflorum were profiled by UPLC-QTOF/MS [16]. Compared with the root of PG in Korea, there were only nine constituents (compounds 5, 31, 76, 79, 83, 91, 94, 95, 97) in common. Meanwhile, the stems and leaves of PG in Korea and in China are both rich in natural components with various structural patterns, including triterpenoid saponins, flavonoids, organic acids, phenols, alcohols, amino acids, coumarins and amino acids, etc., but there are only two similar chemical components (compounds 99, 104). It is also interesting that there are eleven components (compounds 5, 14, 17, 21, 23, 31, 52, 83, 94, 95, 97) reported in stems and leaves of PG in Korea that were found in the root of PG in China. The reason for this phenomenon may be the different analytical methods and the different growing locations.

In this paper, 38 robust known biomarkers enabling the differentiation among root, stem, leaf and seed, were discovered. For the root part, there are 15 potential biomarkers including triterpenoid saponins $(77,79,82,83,89,91,94,95,96,97,101,102,106)$, an organic acid (116) and a phenyl-propanoid (42). For stem part, there are five potential biomarkers including flavonoids (53, 61, 87), a tannin (7) and a triterpenoid saponin (144). For leaf part, there are seven potential biomarkers including flavonoids $(47,59, \mathbf{1 2 5})$, sesquiterpenoids $(\mathbf{1 1 5}, \mathbf{1 1 9})$ and tannins $(\mathbf{2 6}, \mathbf{6 0})$. For seed part, there are 11 potential biomarkers including flavonoids $(8, \mathbf{1 8}, 37,57,69,73,84,99)$, quinones $(55,86)$ and an organic acid (117). These robust biomarkers enabling the differentiation among root, stem, leaf and seed can be used for rapid identification of four different parts of PG grown in northeast China. 
Even so, there are still some unresolved issues. Firstly, pharmaceutical effects associated with these robust biomarkers or these identified compounds should be screened in the future. Additionally, as shown in BPI chromatograms, though 159 compounds were identified there are still many unidentified components. Further research should be carried on based on the formula of these unknown compounds [13]. Most importantly, the stems and leaves of PG should be developed and utilized due to the presence of so many different components from the root. This comprehensive and unique phytochemical profile study revealed the structural diversity of secondary metabolites and the different patterns in various parts of PG. The method developed in this study can be used as a standard protocol for discriminating and predicting parts of PG directly.

\section{Experimental Section}

\subsection{Materials and Reagents}

All samples were harvested from Jilin Province, China, as listed in Table 2, and identified by Professor Ping-Ya Li (School of Pharmaceutical Sciences, Jilin University, Changchun, China). The voucher specimens (No. 2016121-2016144) had been deposited at the Research Center of Natural Drug, School of Pharmaceutical Sciences, Jilin University, Changchun, China. The cultivation ages of the roots are all 2 years, while the others are all 1 year old.

Table 2. Information of samples from Jilin Province, China.

\begin{tabular}{|c|c|c|c|c|c|}
\hline Collection Region & $\begin{array}{l}\text { Mark of } \\
\text { Samples }\end{array}$ & Collection Date & Collection Region & $\begin{array}{l}\text { Mark of } \\
\text { Samples }\end{array}$ & Collection Date \\
\hline \multirow{4}{*}{ Antu County } & S1 & 2 October 2016 & \multirow{4}{*}{ Fusong County } & S4 & 4 October 2016 \\
\hline & L1 & 2 October 2016 & & $\mathrm{~L} 4$ & 4 October 2016 \\
\hline & R1 & 26 October 2016 & & $\mathrm{R} 4$ & 30 October 2016 \\
\hline & D1 & 2 October 2016 & & D4 & 4 October 2016 \\
\hline \multirow{4}{*}{ Hunchun City } & $\mathrm{S} 2$ & 1 October 2016 & \multirow{4}{*}{ Tonghua City } & S5 & 5 October 2016 \\
\hline & L2 & 1 October 2016 & & L5 & 5 October 2016 \\
\hline & $\mathrm{R} 2$ & 27 October 2016 & & R5 & 28 October 2016 \\
\hline & D2 & 1 October 2016 & & D5 & 5 October 2016 \\
\hline \multirow{4}{*}{ Changbai County } & S3 & 30 September 2016 & \multirow{4}{*}{ Jiaohe City } & S6 & 3 October 2016 \\
\hline & L3 & 30 September 2016 & & L6 & 3 October 2016 \\
\hline & R3 & 29 October 2016 & & R6 & 25 October 2016 \\
\hline & D3 & 30 September 2016 & & D6 & 3 October 2016 \\
\hline
\end{tabular}

Acetonitrile and methanol suitable for UHPLC-MS purchased from Fisher Chemical Company (Geel, Belgium). Formic acid for UPLC was purchased from Sigma-Aldrich (St. Louis, MO, USA). Deionized water was purified using a Millipore water purification system (Millipore, Billerica, MA, USA). All other chemicals were of analytical grade. Fourteen standard compounds including platycodin D (111851-201607), mannitol (100533-201304), citric acid (111679-201602), phenylalanine (140676-201405), tryptophan (140686-201303), chlorogenic acid (110753-201716), caffeic acid (110885-201102), dibutyl sebacate (190102-201501), linolenic acid (111631-201605), sucrose (111507-201303), adenosine (110879-201202), monopalmitin (190011-201302), rutin (100080-201610), quercetin (100081-201610), were purchased from the National Institutes for Food and Drug Control (Beijing, China). Seven standard compounds including gallocatechin (201512013), quinine acid (20150321), brusatol (20150410), stigmasterol (20150111), xanthotoxol (20109376), delphinidin (20159567), and atractylenolide III (2014712) were purchased from Beijing Putian Genesis Biotechnology Co., Ltd. (Beijing, China). Nine standard compounds including deapioplatycoside E (160712), deapioplatycodin $\mathrm{D}(160518),-\mathrm{D}_{2}$ (160407), platycoside $\mathrm{E}(160112)$, platycodin $\mathrm{D}_{2}(160721),-\mathrm{D}_{3}(160909)$, platycoside $\mathrm{G}_{3}$ (160921), 2'-O-acetyl-platycodin $\mathrm{D}_{2}$ (160112), 3'-O-acetylplatycodin $\mathrm{D}_{2}$ (160923) were provided by Institute of Frontier Medical Science of Jilin University (Changchun, China). 


\subsection{Sample Preparation and Extraction}

The roots, stems, leaves and seeds of PG from the different sites were respectively air dried, ground and sieved (40 mesh) to give a homogeneous powder. Then $200 \mathrm{mg}$ of the powder was respectively extracted thrice with $80 \%$ methanol at $80{ }^{\circ} \mathrm{C}$ for $3 \mathrm{~h}$ each time. After filtering, the extracts were combined, concentrated and evaporated to dryness. Finally, the desiccated extracts were dissolved and diluted with $80 \%$ methanol to $10.0 \mathrm{~mL}$. The solution was filtered through a syringe filter $(0.22 \mu \mathrm{m})$ and injected directly into the UPLC system. The volume injected was $2 \mu \mathrm{L}$ for each run.

\subsection{UPLC-QTOF-MSE}

The UPLC analysis was performed by a Waters ACQUITY UPLC System. The column used was an ACQUITY UPLC BEH C18 $(100 \mathrm{~mm} \times 2.1 \mathrm{~mm}, 1.7 \mu \mathrm{m})$ from Waters Corporation (Milford, MA, USA). The mobile phases consisted of eluent A $(0.1 \%$ formic acid in water, $v / v)$ and eluent B $(0.1 \%$ formic acid in acetonitrile, $v / v$ ) with flow rate of $0.4 \mathrm{~mL} / \mathrm{min}$ with a liner gradient program: $10 \% \mathrm{~B}$ from 0 to $2 \mathrm{~min}, 10-90 \%$ B from 2 to $26 \mathrm{~min}, 90 \%$ B from 26 to $28 \mathrm{~min}, 90-10 \%$ B from 28 to $28.1 \mathrm{~min}$, $10 \% \mathrm{~B}$ from 28.1 to $30 \mathrm{~min}$. The temperature of the UPLC column and autosampler were set at $30^{\circ} \mathrm{C}$ and $15^{\circ} \mathrm{C}$. Mixtures of $10 / 90$ and $90 / 10$ water/acetonitrile were used as the strong wash and the weak wash solvent respectively.

The MS experiments were performed on a Waters Xevo G2-S QTOF mass spectrometer (Waters Co., Milford, MA, USA.) connected to the UPLC system through an electrospray ionization (ESI) interface. The optimized instrumental parameters were as follows: capillary voltage floating at $2.6 \mathrm{kV}$ $(\mathrm{ESI}+)$ or $2.2 \mathrm{kV}(\mathrm{ESI}-)$; cone voltage at $40 \mathrm{~V}$; source temperature at $120^{\circ} \mathrm{C}$, desolvation temperature at $300{ }^{\circ} \mathrm{C}$ and cone gas flow was $50 \mathrm{~L} / \mathrm{h}$, desolvation gas flow was $800 \mathrm{~L} / \mathrm{h}$. In MSE mode, collision energy of low energy function was set at $6 \mathrm{~V}$, while ramp collision energy of high energy function was set at 20-40 V. To ensure mass accuracy and reproducibility, the mass spectrometer was calibrated over a range of 100-1600 Da with sodium formate. Leucine-enkephalin $(\mathrm{m} / \mathrm{z} 556.2771$ in positive ion mode; m/z 554.2615 in negtive ion mode) was used as the lockmass at a concentration of $200 \mathrm{ng} / \mathrm{mL}$ and flow rate of $20 \mu \mathrm{L} / \mathrm{min}$. Data were collected in continuum mode, all the acquisition of data were controlled by the Waters MassLynx v.4.1 software ( waters, Milford, MA, USA).

\subsection{Data Analysis}

For the screening analysis, the raw data were processed using the streamlined workflow of UNIFI 1.7.0 software (Waters, Manchester, UK) to quickly identify the chemical components [15]. Besides the Waters Traditional Medicine Library in the UNIFI software, a self-built database was created including the information of chemical components from PG based on the literature and on-line databases such as China Full-text Journals Database (CNKI), PubMed, Medline, Web of Science and ChemSpider. Minimum peak area of 200 was set for 2D peak detection. The peak intensity of high energy over 200 counts and over 1000 counts for low energy were the selected parameters in 3D peak detection. A margin of error up to 5 ppm for identified compounds was allowed. Positive adducts containing $+\mathrm{H}$, $+\mathrm{Na}$, and negative adducts including $+\mathrm{COOH}$ and $-\mathrm{H}$ were selected. The verification of compounds was carried out by comparison with retention time of reference standards and characteristic MS fragmentation patterns reported in literature.

For metabonomics analysis, the raw data were processed by MarkerLynx XS V4.1 software for alignment, deconvolution, data reduction, etc. [33]. As a result, the list of mass and retention time pairs with corresponding intensities for all the detected peaks from each data file. The main parameters were as follows: retention time range 0-28 min, mass range 100-1600 Da, mass tolerance 0.10 , minimum intensity $5 \%$, marker intensity threshold 2000 counts, mass window 0.10 , retention time window 0.20 , and noise elimination level 6 . The resulting data were analyzed by principle component analysis (PCA) and orthogonal projections to latent structures discriminant analysis (OPLS-DA). S-plots and VIP-plots 
were obtained via OPLS-DA analysis to find potential biomarkers that significantly contributed to the difference among the groups.

\section{Conclusions}

In the present study, UPLC-QTOF-MSE coupled with UNIFI platform and precise multivariate statistical analyses was used to profile the four parts of PG. For the constituent screening under the optimized conditions, a total of 159 chemical compounds $(73,42,35,44$ compounds characterized from root, stem, leaf and seed, respectively) were identified from PG. The results showed various structural patterns including triterpenoid saponins, organic acids, steroids, phenols, flavonoids, alcohols, amino acids, coumarins, terpenoids, alkaloids and amides. The stem, leaf and seed contain more flavonoids but few saponins that can be easily discriminated from the root.

For the metabolomic analysis, four parts of PG were successfully discriminated into four different clusters. A total of 38 robust biomarkers were discovered. That is to say, 15, 5, 7, and 11 robust biomarkers enabling the differentiation among root, stem, leaf and seed, were characterized. These biomarkers can be suitable for the simultaneous differentiation of four different parts of PG, which is reported for the first time. In a word, these results provided the reliable characterization profiles and the differentiate components among root, leaf, stem and seed of PG grown in northeast China. The method developed in this study can be used as a standard protocol for discriminating and predicting the different parts of PG directly.

Acknowledgments: This work was supported by Talents Team Major Program of Jilin Province of China (JRCBTZ. [2016] No. 3).

Author Contributions: Pingya Li and Jinping Liu conceived and designed the experiments; Cuizhu Wang, Nanqi Zhang and Zhenzhou Wang performed the experiments; Cuizhu Wang, Zeng Qi, Hailin Zhu and Bingzhen Zheng were responsible for data analysis. Cuizhu Wang wrote the paper. Jinping Liu and Pingya Li assisted paper revision.

Conflicts of Interest: The authors declare that they have no conflicts of interest concerning this article.

\section{References}

1. Qi, Y.F. The functional differences in different parts from the same plant. Inf. Tradit. Chin. Med. 1988, 3, $40-42$.

2. Zhang, L.; Wang, Y.L.; Yang, D.W.; Zhang, C.H.; Zhang, N.; Li, M.H.; Liu, Y.Z. Platycodon grandiflorus-An Ethnopharmacological, phytochemical and pharmacological review. J. Ethnopharmacol. 2015, 164, 147-161. [CrossRef] [PubMed]

3. Choi, J.H.; Hwang, Y.P.; Lee, H.S.; Jeong, H.G. Inhibitory effect of Platycodi Radix on ovalbumin-induced airway inflammation in a murine model of asthma. Food Chem. Toxicol. 2009, 47, 1272-1279. [CrossRef] [PubMed]

4. Ahn, K.S.; Noh, E.J.; Zhao, H.L.; Jung, S.H.; Kang, S.S.; Kim, Y.S. Inhibition of inducible nitric oxide synthase and cyclooxygenase II by Platycodon grandiflorum saponins via suppression of nuclear factor- $\mathrm{kB}$ activation in RAW 264.7 cells. Life Sci. 2005, 76, 2315-2328. [CrossRef] [PubMed]

5. Xie, Y.; Pan, H.; Sun, H.; Li, D. A promising balanced Th1 and Th2 directing immunological adjuvant, saponins from the root of Platycodon grandiflorum. Vaccine 2008, 26, 3937-3945. [CrossRef] [PubMed]

6. Jeong, C.H.; Choi, G.N.; Kim, J.H.; Kwak, J.H.; Kim, D.O.; Kim, Y.J.; Heo, H.J. Antioxidant activities from the aerial parts of Platycodon grandiflorum. Food Chem. 2010, 118, 278-282. [CrossRef]

7. Güvenç, A.; Akkol, E.K.; Hürkul, M.M.; Süntar, I.; Keles, H. Wound healing and anti-inflammatory activities of the Michauxia L’Hérit (Campanulaceae) species native to Turkey. J. Ethnopharmacol. 2012, 139, 401-408. [CrossRef] [PubMed]

8. Jeong, C.H.; Shim, K.H. Chemical Composition and Antioxidative Activities of Platycodon grandiflorum Leaves and Stems. J. Korean Soc. Food Sci. Nutr. 2006, 35, 685-708.

9. Liu, D.; Tan, W. Nutritional composition and antioxidant activities of Platycodon grandiflorum flower and leaf. Agric. Food Ind. Hi Tech 2016, 27, 44-46. 
10. Choi, J.H.; Jin, S.W.; Choi, C.Y.; Kim, H.G.; Kim, S.J.; Lee, H.S.; Chung, Y.C.; Kim, E.J.; Lee, Y.C.; Jeong, H.G. Saponins from the roots of Platycodon grandiflorum ameliorate high fat diet-induced non-alcoholic steatohepatitis. Biomed. Pharmacother. 2017, 86, 205-212. [CrossRef] [PubMed]

11. Mazol, I.; Gleńsk, M.; Cisowski, W. Polyphenolic compounds from Platycodon grandiflorum A. DC. Acta Pol. Pharm. 2004, 61, 203-208. [PubMed]

12. Inada, A.; Yamada, M.; Murata, H.; Kobayashi, M.; Toya, H.; Kato, Y.; Nakanishi, T. Phytochemical studies of seeds of medicinal plants. I. Two sulfated triterpenoid glycosides, sulfapatrinosides I and II, from seeds of Patrinia scabiosaefolia FISCHER. Chem. Pharm. Bull. 1988, 36, 4269-4274. [CrossRef] [PubMed]

13. Zhang, F.X.; Li, M.; Qiao, L.R.; Yao, Z.H.; Li, C.; Shen, X.Y.; Wang, Y.; Yu, K.; Yao, X.S.; Dai, Y. Rapid characterization of Ziziphi Spinosae Semen by UPLC/Qtof MS with novel informatics platform and its application in evaluation of two seeds from Ziziphus species. J. Pharm. Biomed. Anal. 2016, 59-80. [CrossRef] [PubMed]

14. Deng, L.; Shi, A.M.; Liu, H.Z.; Meruva, N.; Liu, L.; Hu, H.; Yang, Y.; Huang, C.; Li, P.; Wang, Q. Identification of Chemical Ingredients of Peanut Stems and Leaves Extracts using UPLC-QTOF-MS Coupled With Novel Informatics UNIFI Platform. J. Mass Spectrom. 2016, 51, 1157-1167. [CrossRef] [PubMed]

15. Tang, J.F.; Li, W.X.; Tan, X.J.; Li, P.; Xiao, X.H.; Wang, J.B.; Zhu, M.J.; Li, X.L.; Meng, F. A novel and improved UHPLC-QTOF/MS method for the rapid analysis of the chemical constituents of Danhong injection. Anal. Method 2016, 8, 2904-2914. [CrossRef]

16. Lee, J.W.; Ji, S.H.; Kim, G.S.; Song, K.S.; Um, Y.; Kim, O.T.; Lee, Y.; Hong, C.P.; Shin, D.H.; Kim, C.K.; et al. Global Profiling of Various Metabolites in Platycodon grandiflorum by UPLC-QTOF/MS. Int. J. Mol. Sci. 2015, 16, 26786-26796. [CrossRef] [PubMed]

17. Wang, H.P.; Zhang, Y.B.; Yang, X.W.; Yang, X.B.; Xu, W.; Xu, F.; Cai, S.Q.; Wang, Y.P.; Xu, Y.H.; Zhang, L.X. High-Performance Liquid Chromatography with Diode Array Detector and Electrospray Ionization Ion Trap Time-of-Flight Tandem Mass Spectrometry to Evaluate Ginseng Roots and Rhizomes from Different Regions. Molecules 2016, 2, 603. [CrossRef] [PubMed]

18. Lee, B.J.; Jeon, S.H.; Lee, S.W.; Chun, H.S.; Cho, Y.S. Soil Physico-Chemistry and Saponins Content of Platycodon grandiflorum Radix Cultured from Different Sites in Gyeongnam Province. Korean J. Med. Crop. Sci. 2014, 22, 463-468. [CrossRef]

19. Nguyen, H.; Lee, D.K.; Choi, Y.G.; Min, J.E.; Yoon, S.J.; Yu, Y.H.; Lim, J.; Lee, J.; Kwon, S.W.; Park, J.H. A ${ }^{1} \mathrm{H}-\mathrm{NMR}$-based metabolomics approach to evaluate the geographical authenticity of herbal medicine and its application in building a model effectively assessing the mixing proportion of ilntentional admixtures: A case study of panax ginseng metabolomics for the authenticity of herbal medicine. J. Pharm. Biomed. Anal. 2016, 124, 120-128. [PubMed]

20. Xiao, J.F.; Zhou, B.; Ressom, H.W. Metabolite identification and quantitation in LC-MS/MS-based metabolomics. TrAC Trends Anal. Chem. 2012, 32, 1-14. [CrossRef] [PubMed]

21. Wang, J.R.; Yau, L.F.; Gao, W.N.; Liu, Y.; Yick, P.W.; Liu, L.; Jiang, Z.H. Quantitative comparison and metabolite profiling of saponins in different parts of the root of Panax notoginseng. J. Agric. Food Chem. 2014, 62, 9024-9034. [CrossRef] [PubMed]

22. Rubert, J.; Righetti, L.; Stranska-Zachariasova, M.; Dzuman, Z.; Chrpova, J.; Dall'Asta, C.; Hajslova, J. Untargeted metabolomics based on ultra-high-performance liquid chromatography-high-resolution mass spectrometry merged with chemometrics: A new predictable tool for an early detection of mycotoxins. Food Chem. 2016, 224, 423. [CrossRef] [PubMed]

23. Andersen, M.B.; Rinnan, Å.; Manach, C.; Poulsen, S.K.; Pujos-Guillot, E.; Larsen, T.M.; Astrup, A.; Dragsted, L.O. Untargeted metabolomics as a screening tool for estimating compliance to a dietary pattern. J. Proteome Res. 2014, 13, 1405-1418. [CrossRef] [PubMed]

24. Garcíavillalba, R.; Tomásbarberán, F.A.; Fançaberthon, P.; Roller, M.; Zafrilla, P.; Issaly, N.; García-Conesa, M.T. Targeted and Untargeted Metabolomics to Explore the Bioavailability of the Secoiridoids from a Seed/Fruit Extract (Fraxinus angustifolia Vahl) in Human Healthy Volunteers: A Preliminary Study. Molecules 2015, 20, 22202. [CrossRef] [PubMed]

25. Zhang, P.; Zhu, W.; Wang, D.; Yan, J.; Wang, Y.; He, L. Enantioselective Effects of Metalaxyl Enantiomers on Breast Cancer Cells Metabolic Profiling Using HPLC-QTOF-Based Metabolomics. Int. J. Mol. Sci. 2017, 18, 142. [CrossRef] [PubMed] 
26. Zeng, L.F.; Kong, H.J.; Zhu, M.; Yan, W.D. A facile method to evaluate the quality of Platycodon grandiflorum, A. De Candolle using reference standard extract. J. Funct. Foods 2016, 26, 48-56. [CrossRef]

27. Yoo, D.S.; Choi, Y.H.; Cha, M.R.; Lee, B.H.; Kim, S.J.; Yon, G.H.; Hong, K.S.; Jang, Y.S.; Lee, H.S.; Kim, Y.S.; et al. HPLC-ELSD analysis of 18 platycosides from balloon flower roots (Platycodi Radix) sourced from various regions in Korea and geographical clustering of the cultivation areas. Food Chem. 2011, 129, 645-651. [CrossRef]

28. Ha, Y.W.; Na, Y.C.; Seo, J.J.; Kim, S.N.; Linhardt, R.J.; Kim, Y.S. Qualitative and quantitative determination of ten major saponins in Platycodi Radix by high performance liquid chromatography with evaporative light scattering detection and mass spectrometry. J. Chromatogr. A 2006, 1135, 27-35. [CrossRef] [PubMed]

29. Ferreira, A.C.S.; Monforte, A.R.; Teixeira, C.S.; Martins, R.; Fairbairn, S.; Bauer, F.F. Monitoring Alcoholic Fermentation: An Untargeted Approach. J. Agric. Food Chem. 2014, 62, 6784-6793. [CrossRef] [PubMed]

30. Zou, Z.J.; Liu, Z.H.; Gong, M.J.; Han, B.; Wang, S.M.; Liang, S.W. Intervention effects of puerarin on blood stasis in rats revealed by a ${ }^{1} \mathrm{H}-\mathrm{NMR}$-based metabonomic approach. Phytomedicine 2015, 22, 333-343. [CrossRef] [PubMed]

31. He, J.Y.; Ma, N.; Zhu, S.; Komastsu, K.; Li, Z.Y.; Fu, W.M. The genus Codonopsis (Campanulaceae): A review of phytochemistry, bioactivity and quality control. J. Nat. Med. 2015, 69, 1-21. [CrossRef] [PubMed]

32. Kim, H.J.; Kang, S.H. Ethnobotany, Phytochemistry, Pharmacology of the Korean Campanulaceae: A Comprehensive Review. Korean J. Plant Res. 2017, 30, 240-264. [CrossRef]

33. Zhao, Y.Y.; Cheng, X.L.; Wei, F.; Xiao, X.Y.; Sun, W.J.; Zhang, Y.; Lin, R.C. Serum metabonomics study of adenine-induced chronic renal failure in rats by ultra performance liquid chromatography coupled with quadrupole time-of-flight mass spectrometry. Biomarkers 2012, 17, 48-55. [CrossRef] [PubMed]

Sample Availability: Samples of the compounds are available from the authors.

(C) 2017 by the authors. Licensee MDPI, Basel, Switzerland. This article is an open access article distributed under the terms and conditions of the Creative Commons Attribution (CC BY) license (http://creativecommons.org/licenses/by/4.0/). 